\title{
Vegetation Productivity/Stability and Other Possible Ecological Invariant Relationships Demonstrated in a Microplot Multispecies Pasture Sward
}

\author{
David Scott \\ P.O. Box 115, Lake Tekapo, New Zealand \\ Correspondence should be addressed to David Scott, scottd_hc@xtra.co.nz \\ Received 3 May 2012; Accepted 9 July 2012 \\ Academic Editors: S. Braude and P. Cruz \\ Copyright (C) 2012 David Scott. This is an open access article distributed under the Creative Commons Attribution License, which \\ permits unrestricted use, distribution, and reproduction in any medium, provided the original work is properly cited.

\begin{abstract}
The relationship between vegetation functional characteristics of production, stability and descriptive characteristic of species diversity or dominance, and other possible compositional invariants were investigated in 2400 micro-plot sward of 20 species at randomly assigned positions and using different matrix sizes of adjacent plots and four harvest to derive a combination of "vegetations" of differing species configurations. Within the highest frequency section of the data there was little relationship between productivity and stability (deviance) and either species diversity or dominance (\% contribution of 1st ranked species). However, considering all data, productivity increased with increasing dominance and was unrelated to species diversity, while stability increased with diversity. Of single parameters combining diversity and dominance, the gradient of the log abundance/rank relationship was superior to Shannon $\mathrm{H}$, with both showing productivity increasing with dominance rather than diversity. The fate of individual micro-plots from a mixed species stand through successive harvests was consistent with the $-3 / 2$ thinning rule, though with species cumulative yields. The abundance/rank relationship was compared with many models. Where there was simultaneous fitting to both density and biomass relationships, self-thinning random particle packing models were best and offered an explanation of the process. The positive correlation between regional and local frequency was related to sample area in a random placement model. Observed species composition and mortality was simulated by consideration of plant size related growth and mortality, initial establishment, growth potential and variation in plant size.
\end{abstract}

\section{Introduction}

Vegetation composition is usually discussed in terms of which plant species are present and the particular environment conditions or physiological processes which give competitive or adaptive advantages to some species. However an alternative approach is to consider whether there are properties or characteristics of ecological communities of interacting individuals of the same or different species, as a whole, which lead to some consistencies in composition and function irrespective of the taxonomic species or conditions involved. A number of lines of evidence suggest this may be so.

An overview of many studies in natural, forestry, and agricultural systems is indicating there may be a number of invariants acting in relation to ecosystem function and composition as a whole, which could be regarded as ecological trends, rules, or laws, which operate over many orders of magnitude from cellular metabolism to ecosystems. These include, the near constant final yield of communities in particular environments; the $-3 / 2$ thinning rule between density and size principally demonstrated in plant monocultures, for example, [1-8]; the near linear relationship between the logarithm of relative contribution of a species and it's rank order within a community, for example, [9-20]; the assembly abundance/rank process and models of why that may be so, for example, [21-26]; the inverse relationship between body mass and density in animals and possibly plants, the metabolic rates across diverse taxa scaling to body mass to the $3 / 4$ power, cellular metabolism, and life span to the $1 / 4$ power; mortality to the $-1 / 4$ power, for example, [27-33]; generalities in proximity interactions of individuals in size distributions, growth, and mortality, for example, [34-41]; the positive correlation between the distribution 
of a species and its local abundance, for example, [42, 43]; that the two functional characteristics of ecosystems, their production and stability, related to two different aspects of biodiversity, for example, [44-66].

Each of the generalities has developed almost independently of the others, with each having its own body of evidence, literature, concepts, and methodology, including different groups advocating for different values of the related coefficients according to the supposed mechanisms involved. But all these generalities are in the realm of statistical laws, or bounds in the same manner we commonly assume normal distributions (or in the present context log-normal), in many studies. While each may not be able to make any precise prediction in one-off situations, as for physical or engineering laws, they become prevailing trends as sample frequency increases. The need is to establish what is the linkage and possible mechanisms involved in the interrelationship between these different relationships.

These relationships have as much relevance to agricultural systems as natural systems, and agriculture needs to take cognisance of the generalities, guidelines, or restraints in designing or developing pastoral systems, even if the hope is to find some productive or desirable systems that may be exceptions to the generalities. The present work was an attempt to determine whether these various relationships could be demonstrated in an experimental study at a microscale in the interactions between individuals of a group of pasture species in a sward under a short-term cutting defoliation regime.

In the present study each of the generalities will initially be treated separately in its own "mini-"paper of background and results. The later part of the study attempts to draw together the interrelationship between the generalities from a simulation model. The main feature is that all the interpretations are based on the same set of empirical data so by implication any generalities found must be interrelated also that by repeated use of the same base data in overlapping composite samples of different sizes in preference to strict statistical independence that the datasets are large, albeit for only one combination of environmental conditions and species.

\section{Methods}

2.1. Field Trial. The empirical data were from a small field trial of 2400 contiguous microplots or cells, with each containing one of the treatment combinations from 20 taxa species, 4 seed numbers per microplot $(1,2,3,4$ to vary species potential), 5 blocks of randomized plant positions, 3 further spatial replications, 2 growing environments (separated spatially), with shoot harvesting with microplots on 4 successive monthly occasions. The intention of the design was to give equal initial opportunity and frequency to each of the taxa-species.

The trial was conducted in outdoor conditions at Lincoln, New Zealand, on a cultivated deep moisture retentive Wakanui silt loam. The plot area was further covered with about $1 \mathrm{~cm}$ of steam sterilised potting mix to limit interference from any buried seed on the site. Plastic-coated netting was laid on the area to define both the general plot area and the individual microplot square cells within it, each of $29 \mathrm{~cm}^{2}$ area, with unique coordinates for each cell. The total plot area was $8.2 \mathrm{~m}^{2}$.The seed was from batches of available pasture species (Table 1). Seed was first counted into small plastic centrifuge vials for each treatment combination. The individual species by seed number combinations were then randomly assigned (computer generated) to each of the cells of a $16 \times 5$ cell block. There were 5 such randomisations within adjacent composite blocks. In sowing the trial, it was found more efficient to first position the vials in seed trays of moist sand in the convenience of a laboratory in a mock up of the field plot before a transfer for field sowing. Seeds were pressed into the surface soil. Guard plants of similar treatment combinations, of at least two-cell depth, were sown around the edge of blocks. The two separated growth environments of $16 \times 75$ cells were either $200 \mathrm{~kg} \mathrm{~N}$ $\mathrm{ha}^{-1}$ fertiliser and no irrigation, or no $\mathrm{N}$ fertiliser and weekly spray irrigation. The nitrogen fertiliser rate was chosen to give an estimated similar total growth response to that of the irrigated treatment but attained by a different means. The trial was sown 11 September (Southern Hemisphere spring).

The numbers of established seedlings in each cell were counted on 3-5 December and shoots were harvested on four occasions during summer and early autumn on 1113 December, 8 January, 6 February and 4 March. At each harvest, the plant(s) in each cell was cut at about $1 \mathrm{~cm}$ above ground, transferred to a correspondingly numbered paper towel folder, oven-dried, and weighed. As a cell was defined by the plastic netting boundary, each could be harvested in a single hand-hold.

\subsection{Definitions}

2.2.1. Plant. As the clipped shoot weight of one cell. At harvesting it was impractical to distinguish or separate between any plants from the multiple seeds sown within one cell and they were generally treated as being a single plant.

2.2.2. Species. The units within a vegetation sample can be classified in a number of ways. Those within the present trial were classified in two complementary manners-either as "taxa-species," or as "rank-species" as their rank order in yield within each sample.

2.2.3. Vegetation. As a group of adjacent cells, and done for all $4 \times 4,5 \times 5$ through to $16 \times 16$ cell matrix group sizes as the planting configuration allowed, and done for all overlapping possibilities for each cell.

2.2.4. Productivity. As the mean cell yield within each sample matrix size, expressed as a percentage of the total mean trial yield at each harvest and with the nitrogen environment grand mean scaled to those of the irrigation environment grand mean.

2.2.5. Diversity. The number of different taxa-species present within each matrix size at a particular harvest occasion 
TABLE 1: Taxa-species used, their seed weight, germination, and relative size of plants (cf. grand mean of all plants $=100$ ) prior to first defoliation.

\begin{tabular}{|c|c|c|c|c|}
\hline Code & Taxa-species & Weight (mg) & Germination (\%) & Size $(\%)$ \\
\hline$\overline{\mathrm{A}}$ & Chicory(Cichoriumintybus) & 1.2 & 54 & 516 \\
\hline B & Prairie grass (Bromuswilldenowii) & 11.6 & 87 & 235 \\
\hline $\mathrm{C}$ & Alfalfa (Medicago sativa) & 2.5 & 85 & 310 \\
\hline $\mathrm{D}$ & Plantain (Plantago lanceolata) & 2.2 & 91 & 248 \\
\hline $\mathrm{E}$ & Perennial ryegrass (Lolium perenne) A & 2.3 & 89 & 192 \\
\hline $\mathrm{F}$ & $\mathrm{B}$ & 2.1 & 97 & 158 \\
\hline G & Berseem clover (Trifoliumalexandrinum) & 2.7 & 77 & 123 \\
\hline $\mathrm{H}$ & Red clover (T. pratense) & 3.3 & 93 & 122 \\
\hline I & Sheep'sburnett (Sanguisorba minor) & 8.9 & 74 & 100 \\
\hline $\mathrm{J}$ & Phalaris (Phalaris aquatica) & 1.8 & 47 & 51 \\
\hline $\mathrm{K}$ & Perenniallupine (Lupinus polyphyllus) & 22.3 & 47 & 102 \\
\hline $\mathrm{L}$ & Birdsfoot trefoil (Lotus corniculatus) & 1.2 & 98 & 57 \\
\hline M & Yorkshire fog (Holcus lanatus) & 0.3 & 83 & 51 \\
\hline $\mathrm{N}$ & Alsike clover (Trifolium hybridum) & 1.1 & 96 & 46 \\
\hline $\mathrm{O}$ & Tall fescue (Schedonorus phoenix) & 2.3 & 79 & 39 \\
\hline $\mathrm{P}$ & White clover (Trifolium repens) & 0.5 & 91 & 36 \\
\hline Q & Orchard grass (Dactylis glomerata) & 1.0 & 60 & 34 \\
\hline $\mathrm{R}$ & Caucasian clover (Trifolium ambiguum) & 2.1 & 20 & 51 \\
\hline $\mathrm{S}$ & Lotus (Lotus pedunculatus) & 0.8 & 81 & 27 \\
\hline $\mathrm{T}$ & Sainfoin (Onobrychis viciifolia) & 15.3 & 28 & 13 \\
\hline
\end{tabular}

(realized diversity) was taken as one measure of diversity. The potential number was restricted for the smaller matrix sizes.

2.2.6. Rank. There were two alternative definitions of rank. For "taxa-species" the rank was the rank order of species as determined from their mean yield across all measurements, from the greatest to the least (Table 3). For "rank-species" the total yield for each taxa-species from all cells within each sample of a particular matrix size was determined and expressed as a percentage of the total yield for that sample. A comparison of the percentage contribution within each sample thereafter determined the "rank-species" designation for that sample, again from the greatest to the least.

2.2.7. Dominance. The percentage contribution of the first ranked species in each vegetation sample was taken as one measure of dominance.

2.2.8. Diversity/Dominance. One combined measure of the two aspects of diversity was the gradient $(k)$ of the relationship between log abundance ( $=\log$ percentage contribution) and species rank. This was determined for matrix sizes containing five or more species, but determined using only the first five ranked species. Another was the Shannon $\mathrm{H}$ index derived from the percentage contribution of each of the taxa-species.

2.2.9. Stability, Constancy, or Resilience. Mean deviance between actual and fitted values for each observation in subsequent fitted relationships was used (deviance $=$ $\operatorname{sqrt}\left(\left((\text { actual}- \text { fitted })^{* *} 2\right) /\right.$ actual $\left.)\right)$. As the means of different matrix sizes would implicitly contain a degree of smoothing relating to the number of cells they contained, the individual deviance estimates were scaled up according the square root of the number of cells to make values comparable. For constancy the deviance was for the departure of the individual observations from the mean response relationship, while for resilience it was the departure between the previous harvest and regrowth.

2.3. Data. The matrix size combinations used resulted in 7,488 for $4 \times 4$ through 480 for $16 \times 16$ "vegetation" samples over 4 harvests. While the data for the corner cells in each block was only present once in each of the different sized matrix vegetation samples, that of the central cells was implicitly present in multiple samples of vegetation of a particular matrix size, up to the central cell data being implicit in 64 of the $8 \times 8$ vegetation samples.

Plants within each matrix size were first amalgamated into totals for each taxa-species type (hereafter referred to as "taxa" or "taxa-species"). They were then alternatively reclassified according to their rank order of abundance within that sample, that is, the 1st most abundant, 2nd most abundant, and so forth (hereafter referred to as "rank" or "rank-species"). The two streams of composite datasets were an amalgamation for samples from the different matrix sizes using the two alternative classifications of species type. Note that a particular rank-species position could be occupied by different taxa-species in different samples. The various parameters were fitted separately to each sample from each matrix size.

The use of different matrix sizes and four harvests gave a dispersion of combination of samples having different matrix 
TABle 2: Percentage distribution of observations in combined data for different matrix sizes and species diversity (in 2-species classes) in first and fourth harvests $(n=12376)$.

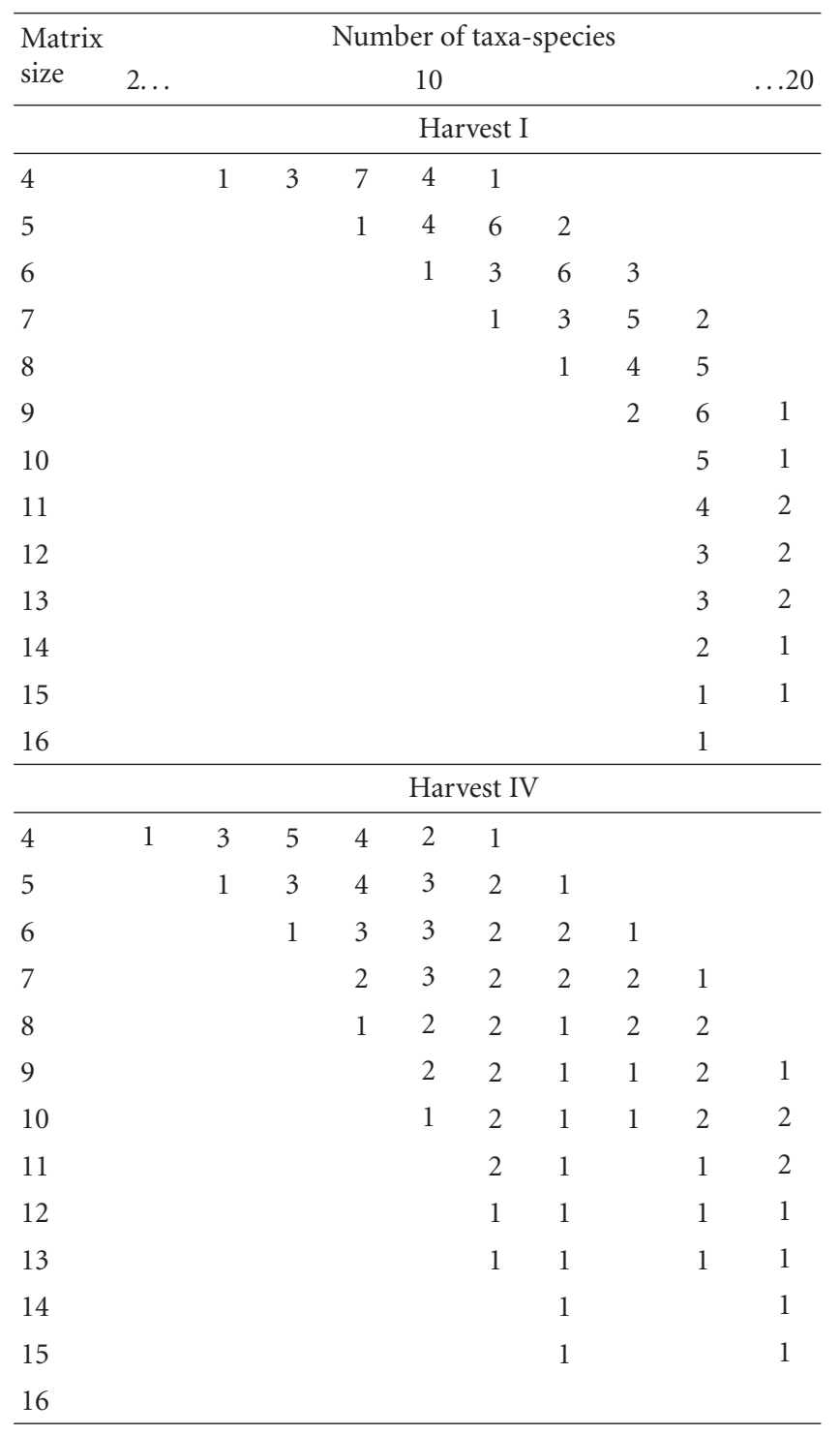

sizes, number of species, and growth (Table 2 ). There were 49,504 observations in the combined dataset. However, there was only a limited range of total species number possible for each matrix size. This was still highly present at the first harvest with the frequency of observations only dispersing to a greater range of combinations with self-thinning by the fourth harvest.

2.4. Analysis. The only truly random element of the trial was the initial allocation of species and seed number within each of the sections of a $16 \times 5$ cell blocks. The subsequently derived vegetation data points were different combinations of adjacent cells with a degree of nonrandomness, confounding and the repeat of the same cell data in different matrix sizes, and repeat in successive harvests.

It was not considered likely that any nonindependence or confounding of samples would influence the investigated
TABLE 3: Mean sward composition (\%) and cumulative mortality (\%) in irrigated and fertilised treatment across four harvests.

\begin{tabular}{lccccc}
\hline \multirow{2}{*}{ Code } & \multirow{2}{*}{ Species } & \multicolumn{2}{c}{ Composition } & \multicolumn{2}{c}{ Mortality } \\
& & Irrig. & $+\mathrm{N}$ & Irrig. & $+\mathrm{N}$ \\
\hline $\mathrm{A}$ & Chicory & 36.5 & 37.6 & 26 & 20 \\
$\mathrm{~B}$ & Prairie grass & 15.7 & 11.8 & 5 & 7 \\
$\mathrm{C}$ & Alfalfa & 7.9 & 12.9 & 30 & 11 \\
$\mathrm{D}$ & Plantain & 10.2 & 10.0 & 17 & 13 \\
$\mathrm{E}$ & Ryegrass A & 7.4 & 5.5 & 14 & 19 \\
$\mathrm{~F}$ & $\quad \mathrm{~B}$ & 6.4 & 4.3 & 5 & 21 \\
$\mathrm{G}$ & Berseem clover & 3.3 & 2.9 & 36 & 38 \\
$\mathrm{H}$ & Red clover & 5.5 & 3.1 & 32 & 20 \\
$\mathrm{I}$ & Sheep's burnett & 2.5 & 2.2 & 26 & 32 \\
$\mathrm{~J}$ & Phalaris & 1.5 & 0.8 & 27 & 44 \\
$\mathrm{~K}$ & Perennial lupine & 1.3 & 0.7 & 60 & 70 \\
$\mathrm{~L}$ & Birdsfoot & 1.0 & 0.9 & 33 & 41 \\
$\mathrm{M}$ & Yorkshire fog & 1.1 & 0.5 & 38 & 59 \\
$\mathrm{~N}$ & Alsike clover & 1.7 & 0.8 & 40 & 54 \\
$\mathrm{O}$ & Tall fescue & 0.5 & 0.4 & 43 & 60 \\
$\mathrm{P}$ & White clover & 1.1 & 0.4 & 41 & 63 \\
$\mathrm{Q}$ & Orchard grass & 0.5 & 0.5 & 42 & 59 \\
$\mathrm{R}$ & Caucasian & 0.1 & 0.2 & 82 & 84 \\
$\mathrm{~S}$ & Lotus & 0.1 & 0.1 & 85 & 87 \\
$\mathrm{~T}$ & Sainfoin & 0.0 & 0.1 & 98 & 98 \\
\hline & & & & &
\end{tabular}

relationships in any systematic biased manner and that there was more value in obtaining as high a number of sample observations as possible to give a full range of possible outcomes with sufficient data density to determine trends. The only formal use of statistical parameters was the correlation coefficient $(R)$ between the raw sample data and fitted function for some of the relationships.

The nonseparation of any multiple plants within a cell would have the effect of increasing the apparent variability in harvested weights of plants of that species. Fitting of response functions used splines with some degree of smoothing to better retain any local variation across the gradient of responses $[67,68]$.

\section{General Taxa-Species Response}

Chicory ( $\mathrm{spA}$ ) was the most successful species in the field trial, with its contribution increasing over successive harvests (Figure 1; Table 3). The species are listed in descending order of their mean contribution and thus give the rank order for taxa-species "suitability" in the particular experimental setting.

The initial equal contribution of species had already become skewed by the first harvest and increasingly more so in subsequent harvests. The trend in composition over time was for a few successful species, with chicory (A) increasing steadily, prairie grass (B) with an initial increase in second and third harvest, and decrease in the last harvest. All other species showed decrease contribution through successive harvests. 


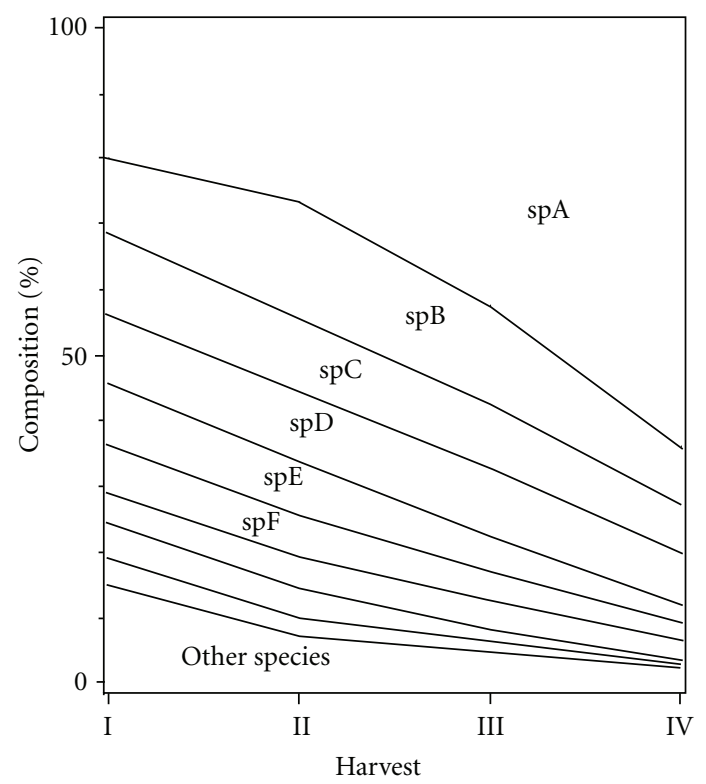

Figure 1: Changes in mean vegetation composition across four successive harvests. Coding as in Table 1.

There was a significant relationship between seed number and microplot yield at first harvest $(R=0.22, P=$ 0.0001 ), but not with seed weight. Indeed with the high success of chicory, with the smallest seed size, the trend was for an inverse relationship with seed weight.

Plants had not been established in about a third of the cells by the first harvest, with further mortality to the second and third harvests, and a larger mortality by the fourth harvest, with only $40 \%$ of the cells remaining occupied. The percentage establishment of some species was higher than their indicated germination rates because of the multiple seed per plot treatment. Using a self-defining definition of "suitability" between species in a particular trial as their relative growth, that for the first harvest was in the order listed. For subsequent harvests, it was the ordering of proportional increase from the previous harvest. For the five most abundant species chicory (A) had the highest suitability across the four harvests, prairie grass (B) suitability decreased, alfalfa (C) increased, and plantain (D) and perennial ryegrass (E and F) remained relatively constant.

The general ordering of the relative success of the different species reflects the generally high fertility, good growing conditions and short term duration of the trial. The pasture species, with the exception of white clover $(\mathrm{P})$, were chosen so that they would remain within their designated microplot for the duration of the trial. Stolon growth of white clover was trimmed to remain within its microplots. Thus competition between species was not for ground area per se, but interaction below ground or between foliages.

There was no great difference between species in their contributions to the sward composition in the two different "environment" treatments. The dominant chicory (A) and alfalfa (C) made slightly greater contribution in the nitrogen-fertilised treatment with the corresponding slightly decreased contribution of other species. The difference between species in relation to treatment "environments" was somewhat greater in relation to mortality, with the largest difference being the lower mortality of chicory (A), alfalfa $(\mathrm{C})$, and red clover $(\mathrm{H})$ in the nitrogen-fertilised treatment.

The results proceed assuming a single guild of species with no large separation of species response according to the two treatment environments.

More details of the changes in species composition and mortality across the four harvests will be given in a later section in association with modelling of those changes.

\section{Species Suitabilities}

4.1. Background. Species differ in their success. The differences are probably relative rather than absolute and relate to a particular group of species within a particular set of environmental conditions. An attempt at the quantification of those relative suitabilities can be derived from a number of sources-from physiological studies of component species leading to estimates of "physiological amplitudes"; comparative field observation in different situations; or the observed outcome of vegetation composition in particular situations (the latter two leading to estimates of "ecological amplitude"). Caution is needed in equating physiological amplitude to ecological amplitude. Observed ecological amplitudes are composed of two components-the physiological responses of each of the component species, and their interaction in an ecological context. In particular, the strong tendency for a community of species to form a log/linear relationship between abundance and rank (to be discussed more fully in subsequent sections) while retaining the relative order of species exaggerates the apparent differences between physiological and ecological amplitudes. This was illustrated in an earlier simpler simulation study showing the gradation in vegetation from gradients of compositions to groups of associations as the strength of a $\log / \operatorname{linear}$ relationship increased [24].

4.2. Results. The relative suitabilities of the different taxaspecies were indicated by their subsequent net growth. The results give two measures. The first is their relative proportions in the resulting vegetation, either as their mean proportions across all harvests, or at each of the harvests (Table 3). However, because those results also probably included the simultaneous interaction of log/linear community process, the relative proportions probably indicate maximum differences in relative taxa-species suitabilities. The alternative measure is just the rank order of the species in terms of their mean proportions (the coding order used in the tests, tables, and figures).

\section{Constant Final Yield}

5.1. Background. One of the observed apparent biological invariants or approximations is the "law of constant final yields." While seldom formally stated, is a generalisation of the observation that, in many situations, experiments or 
trials that different vegetations, within a particular set of environmental conditions, tend to the same similar final yields from diverse origins and compositions. It is prevalent in agricultural species and variety trials. While the objective there is generally to find differences between mixtures, species, or cultivars and is generally so reported, the converse generality is often overlooked of how small those differences can be, that is, how similar total yields were.

There are probably two immediate qualifications, firstly the need to include time so that it is productivity rather than yield (i.e., yield per year); secondly that the constancy refers to each particular set of environmental conditions. The Rothamsted Park Grass experiment [46] is a pasture example of long-term (1862-1991) data showing small fluctuations in annual hay yields around a relatively fixed mean yield, but how that mean is dependent on particular treatments.

The general question is what are the environmental or plant factors that determine that total productivity, and what are the factors that determine the partitioning of that total among different species. For example, Scott et al. [69] have suggested that, for the New Zealand situation, potential pasture production can be estimated from the position of a site along the four environmental gradients of soil moisture (rainfall, soil depth, drainage, irrigation), temperature (latitude, altitude, aspect, and slope), soil fertility (natural or applied), the interaction of animals and plant growing points (grazing, treading), and that for each environmental combination there is a hierarchy of plant species suitabilities. It was also suggested that many species combinations will reach two-thirds to three-quarters of the potential but that only a few species combinations will reach the full potential production for a particular environmental combination.

5.2. Results. The total pasture yields were approximately similar for the four harvests and between the irrigated and $\mathrm{N}$-fertilised treatment (Table 4). Yields were somewhat lower for fourth harvest with a reversal between the two environment treatments. The similarity in total yields justified the scaling of individual sample yields to a common scale of percentage of the grand mean for each harvest, thereby allowing combining the data from the different harvests into a larger composite dataset.

\section{Productivity/Stability}

6.1. Background. A matter of practical implications is the degree to which descriptive characteristics of vegetation or pastures, such as dominance by a few species or the diversity of species, affect their functional characteristics like productivity, stability, or resiliency. One view is that diversity aids production, stability, and resilience-the greater the number of species the greater will be the production, the uniformity of production, and more quickly things will recover from adverse events like defoliation, floods, pests, or fire. This is the prevailing view among the general public, most scientists, and the literature. It makes sense of the broad pattern of the low biodiversity and productivity of arctic and alpine regions thorough to the high biodiversity and production of
Table 4: Observed mean pasture yields $\left(\mathrm{tDMha}^{-1}\right)$ at four harvests. $\mathrm{LSD} 5 \%=0.3$.

\begin{tabular}{lcccc}
\hline \multirow{2}{*}{ Treatment } & \multicolumn{4}{c}{ Harvest } \\
& I & II & III & IV \\
\hline Irrigated & 1.7 & 2.2 & 2.2 & 1.3 \\
N fertilised & 1.4 & 1.9 & 1.9 & 1.5 \\
\hline
\end{tabular}

tropical rainforest, and it is supported by some theoretical studies. But it is extremely hard to find much supporting empirical data, even within the review on biodiversity issues by United Nations Environment Programme [49] and the papers referred to within it, that the generality applies at the level of a particular region, land type, or vegetation. Even a recent consensus review of effects of biodiversity on ecosystem function showed that there are many patterns and that the trends are not strong [62].

However, there is another concept, not well articulated, derived from an overview of agricultural history, some practical studies, for example, [44], and from some theoretical studies. That is, that ecosystem function may be related to two different contradictory aspects of diversity, with production related to how few or dominant one species may be (i.e., tendency to monocultures), and with stability or resilience more related to the diversity of species.

If one takes the view that every farmer in the past and present world was/is a very good experimental ecologist, because their life and livelihood depends on it, then the over-whelming evidence of an overview of the world's agricultural history is that the higher productions come from near monocultures. If there were more productive systems from binary or multispecies systems, then farmers would have long since found them [50]. That is viewing the scene, from the perspective of humans as herbivores and productivity as single plant components. The view is less clear considering humans as carnivores or omnivores with productivity as secondary production of other herbivores, as in grass/legume/other herb/shrub grazing systems.

There is also an intermediate position and evidence of a "hump-backed" relationship of productivity being greatest at moderate level of diversity and decreasing with low or high diversity. The other aspect of the productivity/diversity debate is which is the cause and which is the effect.

There is now an increasing number of experimental studies and meta-analyses of these possible relationships, for example, $[54,55,62,63,65,66]$. The present trial is a further contribution to those comparisons.

6.2. Analysis. For the first part of the productivity/stability section, the combined dataset was summarized into a matrix of number of species and unit classes (1\%) for first ranked species for the scaled productivity estimates. To that was fitted a smoothed bivariate spline (SAS $[67,68]$, proc g3grid (spline, smooth $=0.1$ ), and proc g3d). From that the fitted values for all observations in the base data were estimated and the deviance determined. A similar smoothed bivariate spline was used to indicate the general trends in deviance. 
For the second part of the productivity/stability section, with stability viewed as resilience, as a return to the previous state, productivity was taken as the mean between a pair of successive harvests, stability as the deviance between the two, and the number of species as the mean between successive harvests.

The Shannon $\mathrm{H}$ index and the gradient for the first five species of the log abundance/rank relationship were used as two alternative single-value indices for the dominance/biodiversity relationship.

The third part of the productivity/stability section is the components of the relationships as related to both diversity and the sampling scale. For that the frequency of observations, unit area production, their coefficient of variation, and species variance components were investigated for each matrix size and species diversity size. Though matrix size and diversity class are strictly discrete the results are presented as continuous response contour functions, based on spline smoothing for data cells with greater than twenty observations.

6.3. Results-Rank-Species. The fit between species diversity (number), dominance (percentage contribution of first ranked species), and productivity (relative yield) was only moderate $(R=0.46$ - though highly significant by virtue of high number of observations) (Figure 2). The highest frequency of observations (see graph coding) was for relatively high number of species in the middle ranges for percentage contribution by the first ranked species. Frequency of observation is lower in other combinations, thus less reliable fitting, and theoretical lower limits in some sections (e.g., for four species the first ranked species could not be less than $25 \%$ and still 1 st ranked).

The main trend was for productivity to be related to dominance. The effect of diversity was relatively small, some increase in productivity with increasing species when there is low dominance, a relatively neutral effect over much of the range, and an indication of perhaps an inverse effect where there was high dominance. In the regions where the observational frequencies were highest there are relatively small effects of diversity and dominance on productivity (see figure coding for $\pm 20 \%$ ).

The fit between diversity, dominance, and stability, as expressed by mean deviance, was lower (more variable) than for productivity. The main trend was for stability to increase (= deviance to decrease) as the number of species increased. The indication was for a plateau of relatively stable stability ratings in the centre of the diversity and dominance range, a marked step in relation to diversity, and a decrease at both high and low dominance.

The return to previous composition following disturbance, as determined from successive harvests, to investigate the alternative meaning of stability as resilience, showed similar stability over much of the data field, with stability decreasing (deviance increasing) when there were less than about eight species in a sample (Figure 2(c)).

Combining the number of species and their dominance into two different diversity indices showed that productivity was principally related to species dominance (Figure 3 ). The trend was only conspicuous at the left margin with the increasing dominance by a few species in the later harvests and where observation density was decreasing. While the main trend lines were highly indicative, the variability between individual observations was large (insert graphs) and there was little trend within the main observational data field. The relationship was closer using the initial gradient of the log abundance/rank relationship than for the Shannon $\mathrm{H}$ index.

As indicted earlier the frequency of observation was not evenly spread between different diversity and matrix size combinations (Table 2, Figure 1).

For each matrix size the frequencies were highest towards the higher diversity classes reflecting the initial design of the trial and earlier harvests, with lower diversity combinations occurring at later harvests through self-thinning. The decrease in frequency and diversity at smaller matrix sizes relates to the sampling size scale.

Unit area productivity expressed relative to matrix size and diversity showed highest productivity at maximum species diversity at almost all matrix sizes (Figure 4). There was a general decrease in the $\mathrm{CV}$ (coefficient of variation) of unit productivity for the larger matrix sizes, though a trend for the $\mathrm{CV}$ to be lower for the less productive combinations at the highest matrix sizes. The decrease in CV should not relate to the increase in sampling area per se and is probably more related to the variability between the different combination of species making a diversity matrix size at lower diversity and matrix sizes.

The expression of the results in terms of dominance of a species within samples (\% contribution of 1st ranked species) gives a different perspective. The general trend was for the contribution of the dominant species to increase as the diversity within the sample decreased. This was present at all matrix sizes, though greater at smaller matrix sizes. There would be an element of a mathematical sampling effect if species were supposedly potentially in equal proportions and the loss of one species giving a proportional increase in others. However, the magnitude of the trend is much greater and probably biological with increasing dominance by the more successful species.

Comparison of Figures 2 and 4 suggests that it is the greater effect of the dominance component relative to the diversity effect per se that leads to the more general correlation between dominance and productivity than with diversity.

The variance of the unit productivity relates to the two components, the direct independent effect of species, and the covariance component of the interaction between species on productivity. Both decreased with increasing matrix size as would be expected. The species variance component was high at small matrix sizes and tends to decrease with diversity; was relatively uniform at all diversities at intermediate matrix sizes; and reverses to being higher at intermediate diversities at high matrix sizes. The species covariance was generally negative at lower matrix sizes as would be expected from competitive interactions. They were weakly positive at high matrix sizes and high diversity. 


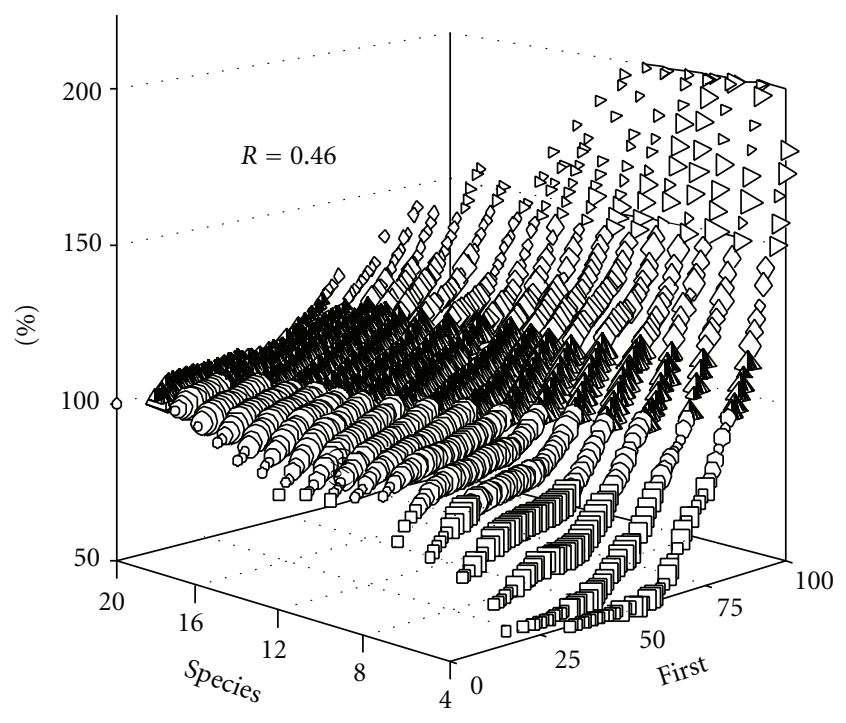

(a) Productivity

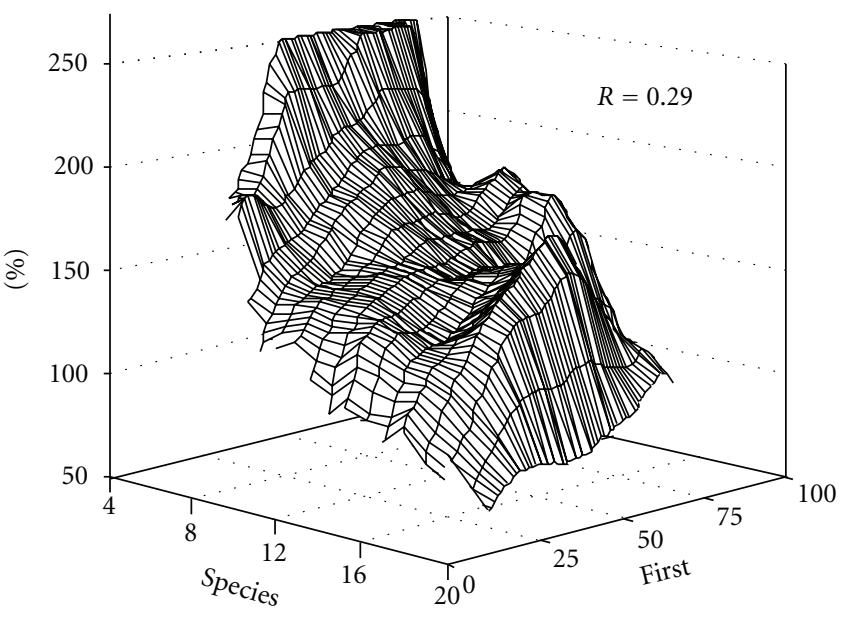

(b) Stability

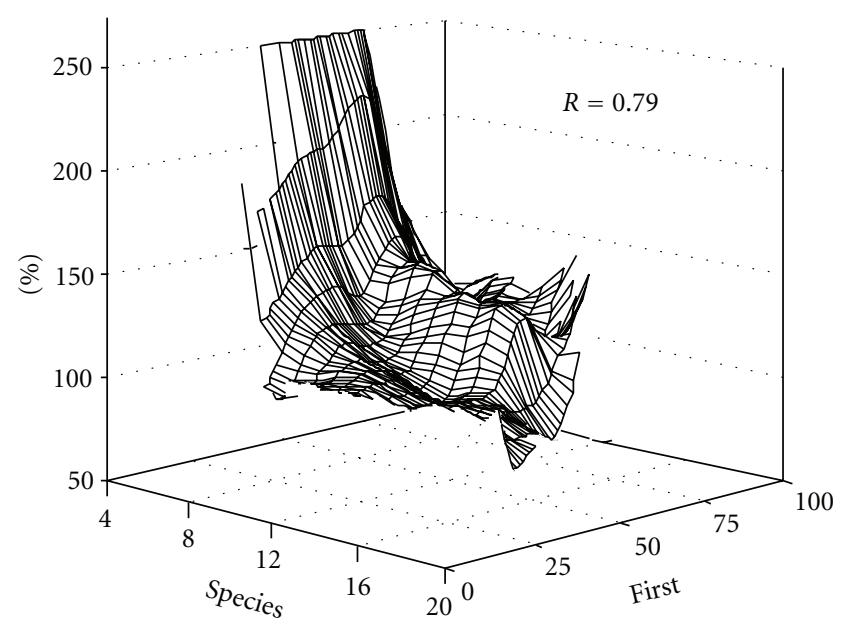

(c) Resilience

FIGURE 2: Relationship between productivity (a), stability (b), and resilience (c) of pasture in respect of the number of species present and percentage contribution of first ranked species. Productivity plotted points coded for both productivity and frequency of observations. Productivity: triangle $=150+\%$, diamond $=120-150 \%$, pyramid $=100-120 \%$, circle $=80-100 \%$, and square less than $80 \%$. Frequency of observations in 5 classes: largest symbol for 100+ observations, then 50-100, 20-50, 10-20, and less than 10. (Note that species number axis is reversed in the stability and resilience graph for ease of presentation).

The scaling factor $(z)$ for the relationship between variance and mean was determined only for samples in which taxa-species were present. The factor was highest (exceeding 2.5 ) in the combinations having the highest frequency of observations and highest productivities. The gradient of changes in the scaling factors was high in many places of the plotted field.

\section{Interaction between Plants}

7.1. Background. There are a large number of possible interactions between specific morphological and physiological characteristics of a particular group of species in a particular environment which result in their differences in abundance and productivity and with many attempts to see generalities in the patterns, for example, [34, 35, 37, 38]. Besides those morphological or physiological differences there has also been the attempt to see if there are any generalities in the interactions irrespective of the species or processes involved (e.g., a general negative correlation between the mass of a plant and the mean mass of surrounding plants $[38,40]$. The present study gave emphasis on the latter type of generalities of which individuals or species thrive and which die.

7.2. Analysis. The influence of surrounding plants on the growth of a particular plant was investigated for the $3 \times$ 3 matrix size from the correlation and stepwise regression analysis between the characteristics and responses of the plant in the central cell with that of the characteristics of the plants in the surrounding eight cells. The correlations were 


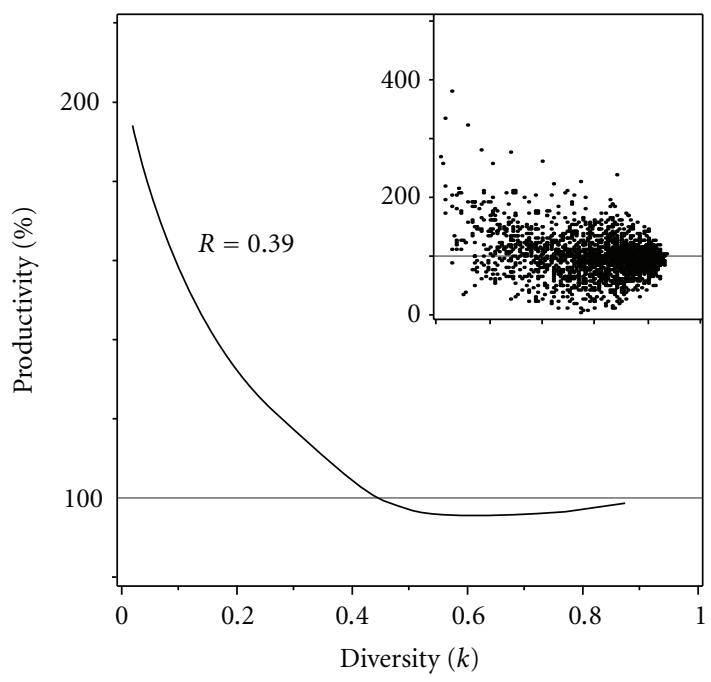

(a)

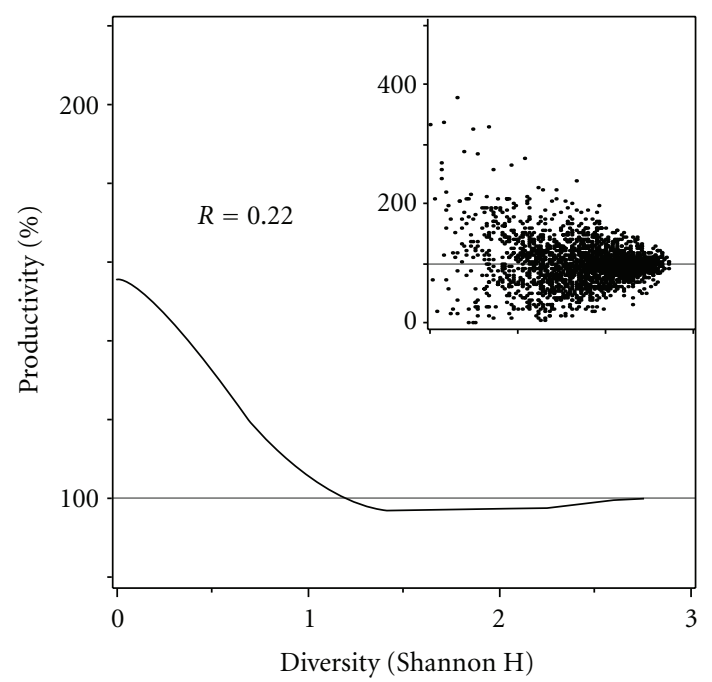

(b)

FIGURE 3: Relationship between productivity (\%) and alternative biodiversity indices using rank-species. For each, on left fitted mean relationship and on right inserted scattergram for a randomly reduced sample (to 5\%) of the same data field. (a). Gradient ( $k$ ) of log abundance/rank graph geometric series fitted to first five species. (b). Shannon H index for available species.

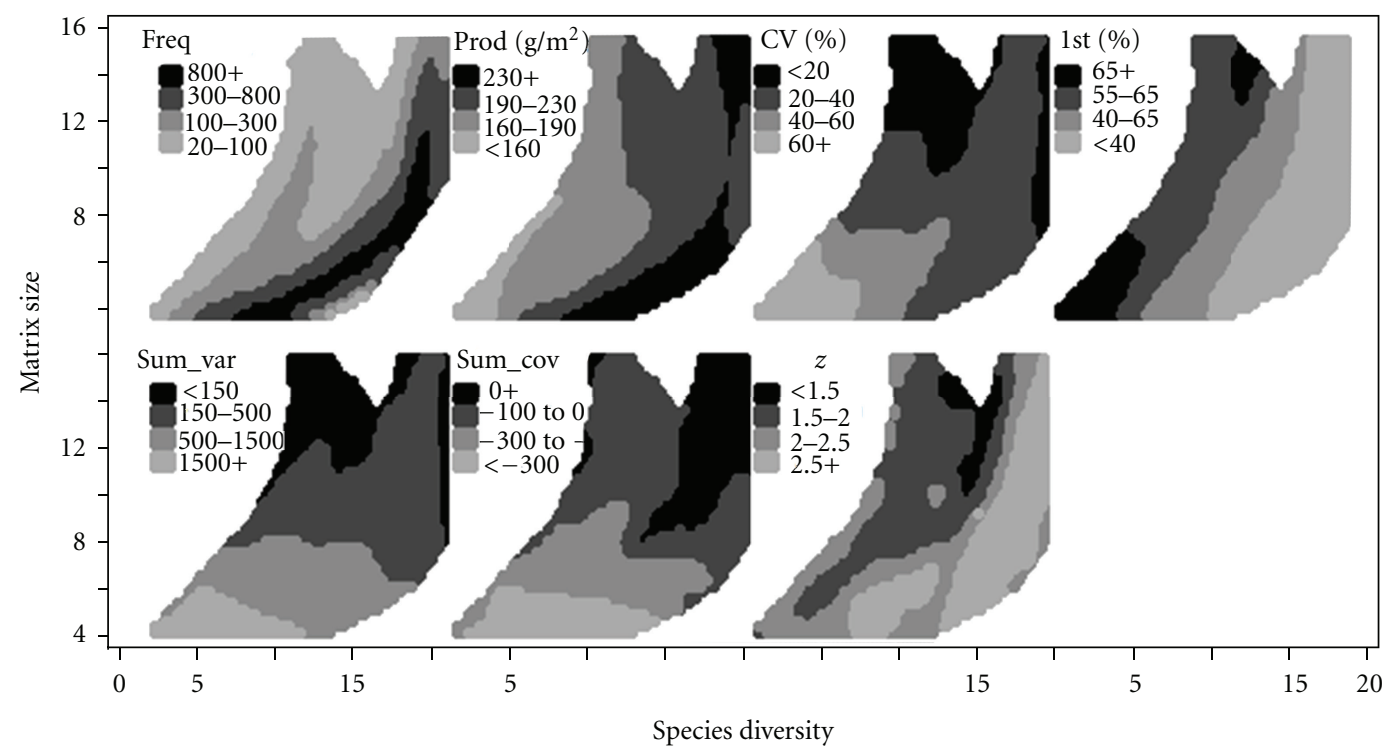

FIGURE 4: Influence of matrix sampling size and taxa-species diversity on components of the productivity/stability relationships within the observational data field. These are frequency of observations in combined dataset; unit productivity ( $\mathrm{g} \mathrm{DM} \mathrm{m}^{-2}$ ); coefficient of variation (CV) of unit productivity; dominance within samples (\% contribution of 1st ranked species); direct species variance component to unit productivity's total variance; species interaction covariance component; scaling factor $(z)$ for the variance/main relationship.

confined to samples in which a central plant was present $(n=$ 404 maximum for each species), with correlations based on relative plant yields, not just presence, so represent possible biological interactions and not fortuitous randomisation of cells.

The factors influencing the mortality of the central plant were similarly investigated for the 2 nd to 4 th harvest using stepwise logistic regression between the fate of the central cell containing plants in a previous harvest and the weight of that plant in the previous harvest, the number of surrounding vacant cells in the current and previous harvests and the combined and separate weights or presence of each other twenty species in the current and previous harvest periods.

The relative frequency of different vacant patch sizes at the four harvests was determined by plotting vacant cells across the whole trial at each harvest and visually assigning them to groups separating by living plants from other such groups.

7.3. Results-Growth Taxa-Species. Considering all central plants, irrespective of species, there was generally a weak 
negative correlation between the yield of the central plant and the combined yield of surrounding plants (Table 5).

There was a general weak negative correlation with the yield of particular surrounding taxa-species, with the exception of a possible weak positive relationship if the surrounding species were sheep's burnett (I) or phalaris (J) (Table 6).

The species are ordered from the most to the least productive. Chicory (A) was the dominant species in the trial. Where it was the central plant there was a negative correlation between its yield and that of the combined yield of surrounding plants and with most individual surrounding taxa-species, but positive if chicory was also one of the surrounding plants. Where other species were the central plant there was the same general negative correlation with the combined yield of surrounding plants, of increasing strength for many species and significant in regression for five species.

Within the body of the correlation table there are indications of a range of specific species interactions. The presence of the same species in the surrounding plants appeared to give positive stimulus to the central plant, for example, sheep's burnett (I), Yorkshire fog $(\mathrm{M})$, tall fescue $(\mathrm{O})$, white clover $(\mathrm{P})$, and lotus $(\mathrm{S})$.

There were a number of reciprocal relationships of whether a species was a central plant or one of the surrounding plants. There were reciprocal negative relationships between chicory (A) and one of the perennial ryegrass cultivars (F), and a similar relationship between chicory and lupin $(\mathrm{K})$ or Yorkshire fog $(\mathrm{M})$. There was a reciprocal positive relationship between the two ryegrass cultivars (E, F) and sheep's burnett (I), between tall fescue $(\mathrm{O})$ and lotus $(\mathrm{S})$, and between white $(\mathrm{P})$ clover, alsike $(\mathrm{N})$, lotus $(\mathrm{S})$ and Yorkshire fog $(\mathrm{M})$.

7.4. Results-Growth Rank-Species. When plants were considered as their rank order in yield within a $3 \times 3$ matrix and for all measurements combined, there was the same weak inverse relationship between the yield of the central plant and the cumulative yield of surrounding plants as for the taxa-species subdivision.

However, when the data was subdivided according to the rank of the central plant relative to the rank of surrounding plants, for each possible ranking of the central plant there was a general positive moderate correlation between its rank and cumulative yield of surrounding plants. The comparison was only taken to fourth ranked plant since only eight ranks were possible and in many samples the later ranks represented zero yields due to plant mortality.

In the subdivisions for different rankings of the central plant there was generally a high positive correlation with immediately adjacent plants of similar ranking and lessening for more remotely ranked plants.

7.5. Results-Mortality Taxa-Species. For mortality, the greatest frequency of highly significant effects for both individual species and combined results (21) was the inverse relationship between the mortality of a plant and its weight in the previous harvest (14/21), a direct relationship with
TABLE 5: Correlation $(r \times 100)$ between the yield of the central plant rank (if present) in $3 \times 3$ matrix and mean rank of surrounding eight plants.

\begin{tabular}{lccccc}
\hline \multirow{2}{*}{ Central plant } & \multirow{2}{*}{ Sum } & \multicolumn{4}{c}{ Surrounding plant rank } \\
& & R1 & R2 & R3 & R4 \\
\hline ALL & -13 & 36 & 19 & 8 & 2 \\
R1 & 29 & na & 38 & 20 & 5 \\
R2 & 47 & 37 & na & 55 & 32 \\
R3 & 43 & 21 & 56 & na & 72 \\
R4 & 23 & -3 & 30 & $\mathbf{6 1}$ & na \\
\hline
\end{tabular}

the number of vacant adjacent cells (11/21), and a direct relationship with harvest (10/21). There were also a number of species specific interactions between the mortality of the central plant and the weight of a specific surrounding species either in the previous period (11/21) or current harvest period (7/21) but not with their combined weight.

The general inverse relationship between the mortality of a plant and its previous size will be dealt with more fully in a subsequent section.

Mortality of the central plant was greater with greater vacant surrounding cells (less surrounding plants) (Table 7). There was also the coalescing of smaller vacant patches into larger vacant patches over successive harvests (Table 8).

7.6. Discussion. The observations in the dataset analysed were derived from overlapping measurements of the same raw data, that is, what was a central cell in one observation became part of the surrounding cell set in another, and visa versa. In that sense there were reciprocal relationships between the interactions described.

In the growth of taxa-species the basis of such specific species interactions (e.g., legume nitrogen fixation or allelopathy), was not part of this investigation, but the occurrence of such specific interactions was noted, that is, the general weak negative correlation between the central plant and the sum of surrounding plants, and some specific taxa species interactions. The correlation matrix is similar to a covariance matrix, and for the sample matrix size used the mean values were negative. The probability of such negative correlations or covariances probably should be considered as part of any simulation of species dynamics forming vegetation compositions.

It is not clear whether any of these trends represent ecological insights of plant interactions. The meaning of positive correlation between the central plant and the mean of surrounding plants when expressed as rank is not clear. It is possible just saying that if it was generally a high yielding $3 \times 3$ matrix samples that all the component plants were higher yielding indicating a degree of mutualism rather than competitive replacement. The trends might also be just a consequence of ranking data.

The greater mortality of the central plant with a greater number of vacant surrounding cells seems counterintuitive 
TABLE 6: Correlation $(r \times 100)$ between the yield of the central plant (if present) in $3 \times 3$ matrix and the mean of surrounding eight plants. Taxa-species coding as in Table 1 . Positive correlations shaded, c 5\% significance bold, c1\% significance bold italic, and first four factors in stepwise regression underlined.

\begin{tabular}{|c|c|c|c|c|c|c|c|c|c|c|c|c|c|c|c|c|c|c|c|c|c|}
\hline \multirow{2}{*}{$\begin{array}{l}\text { Central } \\
\text { plant }\end{array}$} & \multirow{2}{*}{ Sum } & \multicolumn{20}{|c|}{ Surrounding taxa-species } \\
\hline & & A & B & $\mathrm{C}$ & $\mathrm{D}$ & E & $\mathrm{F}$ & G & $\mathrm{H}$ & I & $\mathrm{J}$ & $\mathrm{K}$ & $\mathrm{L}$ & M & $\mathrm{N}$ & $\mathrm{O}$ & $\mathrm{P}$ & Q & $\mathrm{R}$ & S & $\mathrm{T}$ \\
\hline ALL & -13 & -6 & -8 & -1 & -3 & -8 & 0 & -5 & -4 & 3 & 1 & -3 & -2 & -6 & -2 & -2 & -3 & -3 & -2 & -2 & -2 \\
\hline A & -15 & 4 & -7 & -2 & $\underline{-11}$ & -9 & -19 & -8 & -2 & -14 & -3 & -17 & -9 & -17 & -3 & -3 & -8 & -12 & 6 & -2 & . \\
\hline B & -7 & $\underline{-15}$ & 4 & 1 & 9 & 3 & 2 & -6 & 13 & 6 & -8 & 2 & -4 & 4 & 1 & -2 & -4 & -1 & -14 & -11 & -1 \\
\hline C & -21 & -25 & $\underline{12}$ & -13 & 10 & -17 & 9 & 13 & $\underline{-1}$ & -2 & -4 & 12 & $\underline{13}$ & 15 & -6 & -14 & 18 & 2 & $\underline{9}$ & $\underline{3}$ & -5 \\
\hline D & -30 & -17 & -11 & -11 & -14 & -11 & -1 & 0 & -6 & -11 & 1 & -3 & 0 & 8 & 1 & 0 & -2 & $\underline{20}$ & -8 & -7 & -4 \\
\hline E & -8 & -4 & -13 & 1 & -6 & -5 & -8 & -12 & -3 & $\underline{20}$ & 5 & -8 & 29 & 14 & 14 & 6 & -11 & 9 & 0 & -7 & -3 \\
\hline $\mathrm{F}$ & -19 & $\underline{-19}$ & -4 & 13 & -21 & 21 & -6 & $\underline{13}$ & 7 & $\underline{19}$ & -9 & 8 & -11 & $\underline{5}$ & -1 & -5 & 0 & 11 & -10 & 10 & 0 \\
\hline G & -9 & -9 & 1 & -3 & 1 & -9 & 7 & 1 & 1 & -6 & 2 & 2 & -10 & -6 & -2 & 4 & -4 & 4 & -4 & -4 & -6 \\
\hline $\mathrm{H}$ & -10 & -10 & 0 & -10 & -3 & 3 & $\underline{7}$ & 0 & . & $\underline{8}$ & 11 & 8 & -2 & 0 & -2 & $\underline{23}$ & 4 & 1 & 8 & 2 & -3 \\
\hline I & -10 & -20 & 0 & -7 & -7 & $\underline{22}$ & $\underline{27}$ & 2 & 18 & $\underline{17}$ & 0 & 15 & 5 & 12 & 7 & 8 & $\underline{14}$ & -2 & 11 & 16 & -1 \\
\hline $\mathrm{J}$ & 2 & -9 & 9 & -5 & $\underline{22}$ & -16 & 15 & $\underline{14}$ & -7 & $\underline{13}$ & -8 & 17 & 10 & -7 & -10 & 4 & $\underline{24}$ & 0 & 1 & -2 & 1 \\
\hline K & -13 & -21 & 0 & 5 & -4 & -2 & 6 & 3 & 10 & 10 & 11 & $\underline{13}$ & -1 & $\underline{20}$ & 14 & $\underline{20}$ & 17 & -9 & 12 & 8 & . \\
\hline $\mathrm{L}$ & -17 & -19 & -3 & 3 & -10 & 8 & -5 & 7 & -5 & $\underline{14}$ & $\underline{18}$ & 3 & 9 & 9 & 9 & 0 & 4 & -3 & $\underline{15}$ & -5 & \\
\hline $\mathrm{M}$ & -22 & -18 & -12 & -10 & -8 & 0 & -13 & -2 & 8 & -14 & -15 & 7 & $\underline{7}$ & $\underline{34}$ & -10 & 9 & 15 & 3 & 5 & -3 & 1 \\
\hline $\mathrm{N}$ & -20 & -14 & -1 & -20 & -6 & 3 & -7 & -1 & -10 & 12 & -12 & -8 & -3 & -9 & -7 & 10 & $\underline{52}$ & 8 & 2 & -5 & . \\
\hline $\mathrm{O}$ & -2 & -6 & -11 & -3 & 17 & -9 & -6 & 12 & 8 & 0 & 6 & 8 & 2 & 9 & $\underline{15}$ & $\underline{19}$ & 25 & 5 & . & 75 & -1 \\
\hline $\mathrm{P}$ & -20 & -26 & 0 & $\underline{6}$ & -9 & -10 & -6 & -8 & -2 & $\underline{-3}$ & -1 & 6 & -2 & 22 & $\underline{44}$ & 1 & -5 & -6 & 14 & 5 &. \\
\hline Q & -6 & -13 & -2 & -5 & 10 & -9 & 2 & $\underline{16}$ & -1 & 5 & 1 & -1 & -10 & -2 & 7 & $\underline{12}$ & 0 & -9 & 7 & 10 & . \\
\hline $\mathrm{R}$ & -27 & -18 & -15 & -10 & -18 & $\underline{6}$ & -8 & 4 & 0 & 0 & $\underline{39}$ & 4 & 2 & 19 & -1 & . & 3 & $\underline{24}$ & . & $\cdot$ & 0 \\
\hline$S$ & -13 & -6 & -14 & -10 & -10 & -16 & -5 & 15 & -11 & 0 & -5 & -9 & -3 & $\underline{-10}$ & -12 & $\underline{81}$ & $\underline{19}$ & -4 & $\cdot$ & 26 & 11 \\
\hline $\mathrm{T}$ & -37 & . & 5 & -9 & 5 & -18 & -3 & $\underline{-4}$ & -36 & -10 & $\underline{-9}$ & . & . & -9 & . & -27 & . & . & 75 & $\underline{97}$ & $\cdot$ \\
\hline
\end{tabular}

TABLE 7: Mortality (\%) of plants present at a previous harvest as related to the vacancy of surrounding cells $(n=3938)$ (for combinations with greater than 20 plants).

\begin{tabular}{lccccccccc}
\hline \multirow{2}{*}{ Harvest } & \multicolumn{10}{c}{ Vacant adjacent cells } \\
& 0 & 1 & 2 & 3 & 4 & 5 & 6 & 7 & 8 \\
\hline II & 2 & 3 & 7 & 6 & 7 & 7 & 8 & & \\
III & 11 & 8 & 17 & 15 & 10 & 17 & 20 & & \\
IV & & 11 & 26 & 24 & 28 & 30 & 37 & 44 & 39 \\
\hline
\end{tabular}

TABLE 8: Frequency of different vacant patch sizes (number of adjacent cells) at successive harvests.

\begin{tabular}{lcccc}
\hline \multirow{2}{*}{ Harvest } & \multicolumn{4}{c}{ Patch size } \\
& $1-4$ & $5-10$ & $11-20$ & $21+$ \\
\hline I & 237 & 39 & 0 & 0 \\
II & 223 & 39 & 9 & 0 \\
III & 185 & 43 & 21 & 0 \\
IV & 79 & 43 & 23 & 16 \\
\hline
\end{tabular}

in that it might have been expected that with lesser competition from surrounding plants there might have been lesser mortality of the central plant.

The explanation is possibly in the confounding of the continual self-thinning mortality over successive harvests (particularly the last harvest) and the consequent coalescing of smaller vacant patches into larger vacant patches (Table 8). The random sowing design of the trial did give a high frequency of separated small vacant patches from either non-establishment or early mortality by the first harvest. Random mortality during subsequent periods led to coalescing of adjacent small patches into fewer larger patches. A consequence in subsampling to form $3 \times 3$ cell matrix with a living central plant is of increasing probability of vacant surrounding cells. An ecological interpretation would be of patch formation from local environmental variability or depletion, with the effect on all plants within an area.

\section{Self-Thinning}

8.1. Background. Another apparent invariant is the $-3 / 2$ selfthinning law of the inverse relationship between density and the weight of remaining plants in stands of initially high number of potential propagules. Empirical evidence suggests that the rule applies over a 6-fold difference in plant density and 9-fold differences in plant dry weight, from a range of different vegetations, with little variation in gradient and intercepts (Figure 2 in Westoby [2], reproduced from White [1]). Those relationships have been largely established in plant monocultures or in forestry where other species make minor contribution relative to the dominant species and that the relationship relates to some function of either plant volume to ground area ratio, or allometric growth relationships 
within plants. There is some experimental evidence that "the rule does apply to mixed-species stands taken as a whole, but not to each component taken separately" [2]—p186, [3]. This relationship has been principally investigated for plants at small to moderate area scales.

It is noted that the sum of the reciprocal gradient $(-2 / 3=$ $-0.67)$ and the gradient of the abundance/rank relationship for a mature grassland $(-0.32)$ is close to unity.

The same possible relationship has been almost independently studied in animals at moderate to large geographic area scale on the relationship between animal species weight or biomass and their population density $[6,27]$. Again there is an inverse logarithmic relationship. As those reviewers say the "regression slopes for the abundance/body mass relationship tend to cluster around $-0.75(\mathrm{sic}-3 / 4)$ although the scatter is wide and the mean slope is -0.51 ," and their Table 1 for different animal groups shows variation between -0.43 and -0.86 . In domestic stock the value of about $0.72-$ 0.75 is used as the exponent for relationship between "liveweight" and "metabolic live-weight." Ernest et al. [6] have noted that "the average use of a plant of a given body size was 1.5 orders of magnitude higher than those of equivalent sized mammals."

8.2. Analysis and Results. The relationship was examined for the $8 \times 8$ matrix size using either the shoot weight of plants at each harvest, or their cumulative weight to that harvest. The scattergram of density and cumulative weight of remaining plants showed regions of high observational density corresponding to the four harvests (Figure 5). The lower right corresponds to the first harvest and successive higher groups with successive harvests, with the upper left the last harvest and extending into the third group. The data has a limit of possible densities on the right margin of one plant per cell for the $2 \mathrm{nd}$, 3rd, and 4th harvest with these discrete possible densities discernable in the plotted points. The first harvest data (lower right) estimated weight and density according to the seedling plant density one week previously. In subsequent harvest the material within a cell was regarded as a single plant.

The trend within 2nd, 3rd, and 4th harvests was for the cumulative weight of residual plants to be increasing and their density decreasing at a rate within the bounds of the $-3 / 2$ thinning rule.

The trends were examined for each of the matrix sizes and for the mean, maximum, cumulative mean, and cumulative maximum weights within each sample. The groups were most bunched, discrete, and within the central bounds for cumulative mean weights. The trend in gradients was similar for other configurations, below the bounds for mean weights, and above the bounds for maximum and cumulative maximums and dispersion more scatted for all configurations. The dispersion reduced with increasing matrix size.

Within the $8 \times 8$ matrix size, the gradient between the third and fourth harvests for the same sample averaged -1.60 with standard deviation of 0.92 , and the inverse gradient of -0.72 and standard deviation of 0.22 .

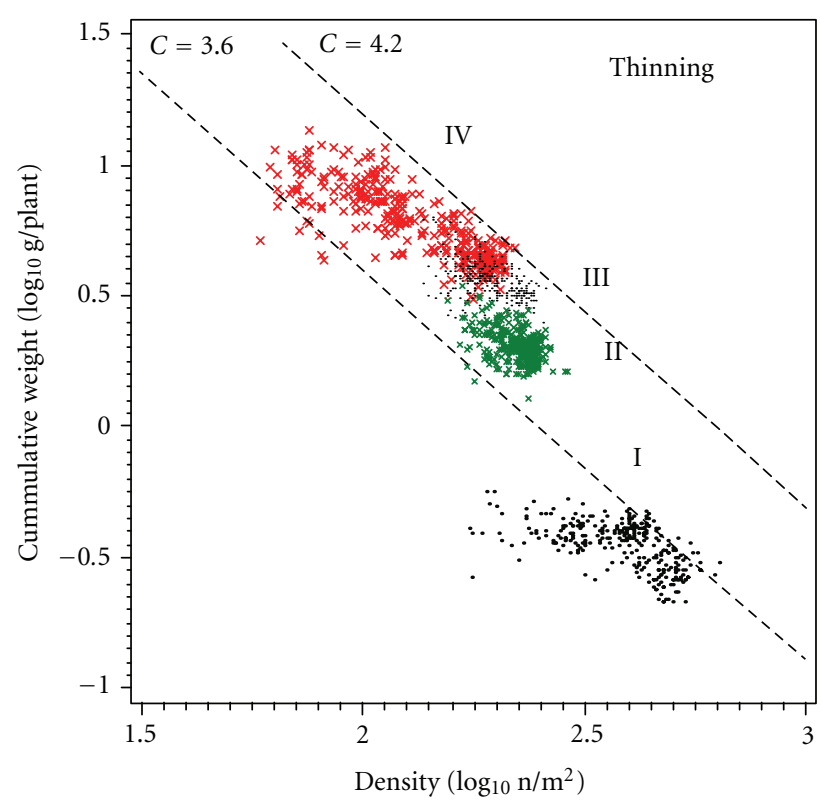

FIGURE 5: Scattergram between cumulative relative weight of remaining plants and their density over four successive harvests (randomly reduced to 5\%) compared to a band for the $-3 / 2$ thinning relationship. Harvest 1 = black, II = red, III = black, and $\mathrm{IV}=$ green .

There was no relationship between the gradient and the $\log /$ linear diversity index $(k)$ of the sample from which they came.

The gradients within samples for a particular harvest departed markedly from -1.33 , being $-0.27,-0.37,-0.41$, and -0.66 for the 1 st to 4 th harvest, respectively.

Considering all matrix sizes, the correlation between the number of plant present within a sample and log mean productivity decreased with increasing matrix size (not presented). Correlations were similar for linear, square root and cube root transformations of number of plants present. A discussion of the results will be part of the General Discussion.

\section{Abundance/Rank Distribution}

9.1. Background. Another one of the observed apparent ecological invariants is the relative constancy of species proportions in different communities. A common feature is the approximately linear relationship between logarithm of relative contribution of species and their rank order within a community.

These have been principally determined from plant and animal studies in natural communities, for example, reviews $[9,11,15,21-23]$. Another line of evidence for this abundance/rank relationship is the dry-weight-rank method of pasture analysis [10]. Implicit in that method is the observation that vegetations, at the microscale of the sample quadrat, have the three most abundant species in the ratio of $70.2: 21.1: 8.7$; proportions at a macroscale are made up of combinations of species and micro-areas in those ratios. The near constancy of these ratios has 
been supported by empirical observations from a range of grasslands [12, 14, 16-19]. With most improved pastures dominated by either one or only a few species, the general gradient of the line is steep relative to more diverse natural communities. For mature grasslands the mean value of the regression coefficient $(k)$ is between abundance and rank is about -0.32 .

I introduced the concept of a need for a conformity index to describe how closely the observed distribution follows a strict $\log /$ linear relationship [24]. The correlation coefficient was not considered appropriate as it only measures the correspondence in the trends and will always be high and negative for retrospectively ranked data. The need is for an index constant for a group of species in one situation, as the mean proportional adjustment needed to make observed values corresponds to a strict $\log /$ linear relationship.

9.2. Analysis and Results. The mean proportional contribution of rank-species was determined across each matrix size and harvest. Considering all species across all matrix sizes, the mean data showed an approximate linear relationship between the rank order of species and the logarithm of their abundance. That relationship varied with harvest, matrix size, and the number of species within a matrix size.

The changes in gradient partly reflect the design of the trial with the near initial equal opportunity for all species, implying $k=1.0$. The value had already decreased to a mean value of 0.81 by the first harvest and continued to decrease through successive harvests with self-thinning and increased dominance (Table 9, Figure 6). The mean percentage of the first ranked species increased from $26 \%$ to $58 \%$.

The same effect of time was present when considering the number of species present within different matrix sizes from potentially a high number of species in the earlier harvest to fewer in later harvests. The insert shows that there was large variation between individual observations.

The earlier sections have mainly dealt with the proportional contribution of the first ranked species as a measure of dominance and introduced the coefficient $(k)$ as the gradient of the line or the coefficient of the geometric series representation of the data as a more general parameter using further species.

Considering first the general relationship between abundance and rank the study used both rank- and taxa-species. There was a high correlation in the coefficient $(k)$ whether based on either rank- or taxa-species but the mean gradient differed from exact correspondence (Figure 7-reference line). In samples with steep relationship between abundance and rank (low coefficient values) the gradients were steeper (abundant species more abundant) based on rank-species than from the general rank order of taxa-species. By contrast in samples with more even distribution of abundances (high coefficient) there was wide difference between the two estimates which was principally related to plants of the more highly ranked taxa-species being small or absent from some samples.

9.3. Discussion. The empirical data did show the approximately linear relationship between log abundance and rank.
TABle 9: Variation in fitted coefficient $(k)$ for the first five species for the geometric series description of relative abundance/rank relationship as related to available species number, matrix size, and harvest.

\begin{tabular}{lcccc}
\hline & \multicolumn{4}{c}{ Number of species } \\
& 20 & 15 & 10 & 5 \\
\hline \multirow{2}{*}{ Harvest } & 0.82 & 0.74 & 0.65 & 0.44 \\
\hline I & $4 \times 4$ & \multicolumn{4}{c}{ Matrix size } \\
II & 0.61 & $0 \times 8$ & $16 \times 16$ \\
III & 0.53 & 0.78 & 0.82 \\
IV & 0.44 & 0.72 & 0.75 \\
\hline
\end{tabular}

The gradient could be interpreted as being strictly log/linear. The relationship tended to change from the log normal form to a more linear form as the number of species in a sample decreased (Figure 6). Both will be discussed further in the next section in comparison with various other models of the relationship.

The dry-weight-rank pasture literature suggests as value for $k=0.32$ for mixed grasslands. The present results were only approaching that in later harvests (Table 9). A much higher value prevailed throughout most of the period.

\section{Abundance/Rank Models}

10.1. Background. Several classes of models have been developed to describe the observed change in abundance on the macroscale when species are ranked retrospectively from the most to the least abundant and by implication the supposed ecological processes involved [20,41].

The first type of assembly models is those of some general mathematical form but having parameters that have to be estimated from the empirical data at hand. These include the geometric series (finite or infinite number of species), truncated log normal, and the log series as described by others. The simplest may be a polynomial regression with no implied functional interpretation (here a quadratic fitted to the ten most abundant species).

The concept of the geometric series model for species colonizing a site is of a first species supposedly preempting some fraction $(1-k)$ of a total niche, a second species a fraction $k$ of the remaining niche, the third species the same fraction of the remainder, and so forth, for a finite or infinite number of species. The attraction of the model is its simplicity, that it generally gives a reasonable to good description of empirical data, has only a single parameter, the relationship can be fitted directly to empirical data, and for field use, the parameter can be estimated directly from either the proportion of the most abundant species or from the ratio of abundance of any two species. I used visual assessment of the later to modify the dry-weight vegetation ranking technique [70]. In those senses it is a useful standard for comparing different models. Its disadvantage is that it does not offer any functional analogue of why the reduction in proportion should be constant for successive species. 


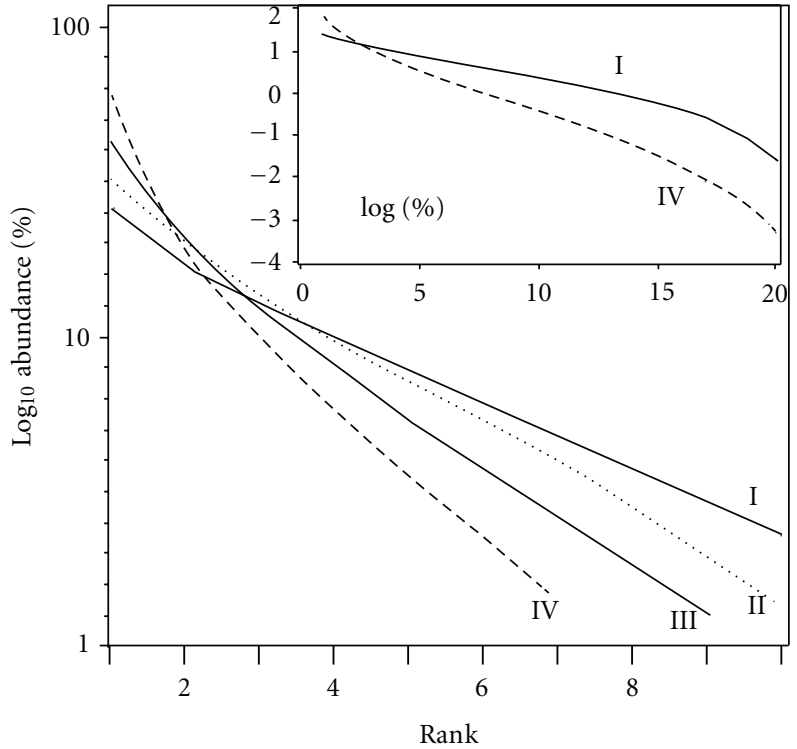

(a) Harvest

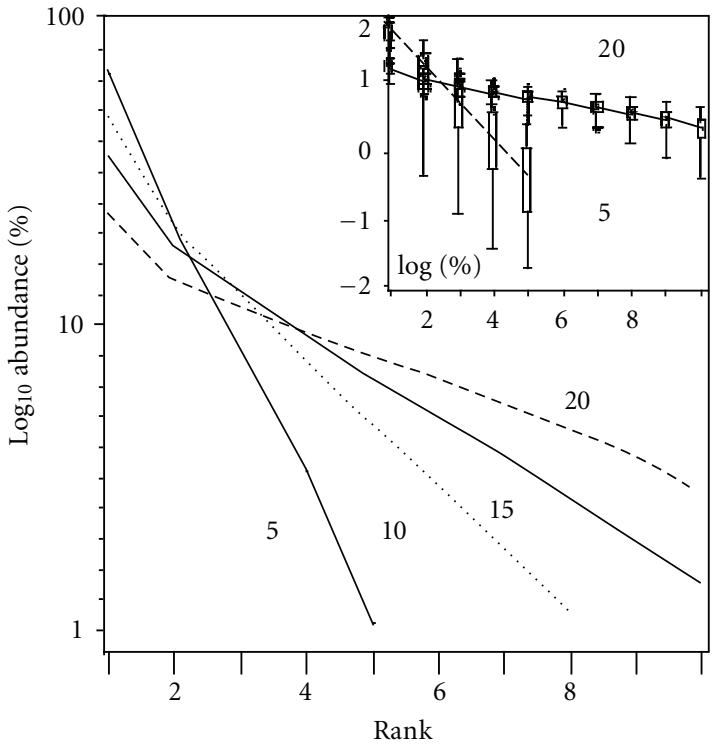

(b) Number of species

FIGURE 6: Mean relationship between abundance and rank order of species across all matrix sizes. (a) Examples for four successive harvests for ten species with insert for twenty species for first and last harvest. (b) Examples for groups with different number of species, with insert of reliability for two of them.

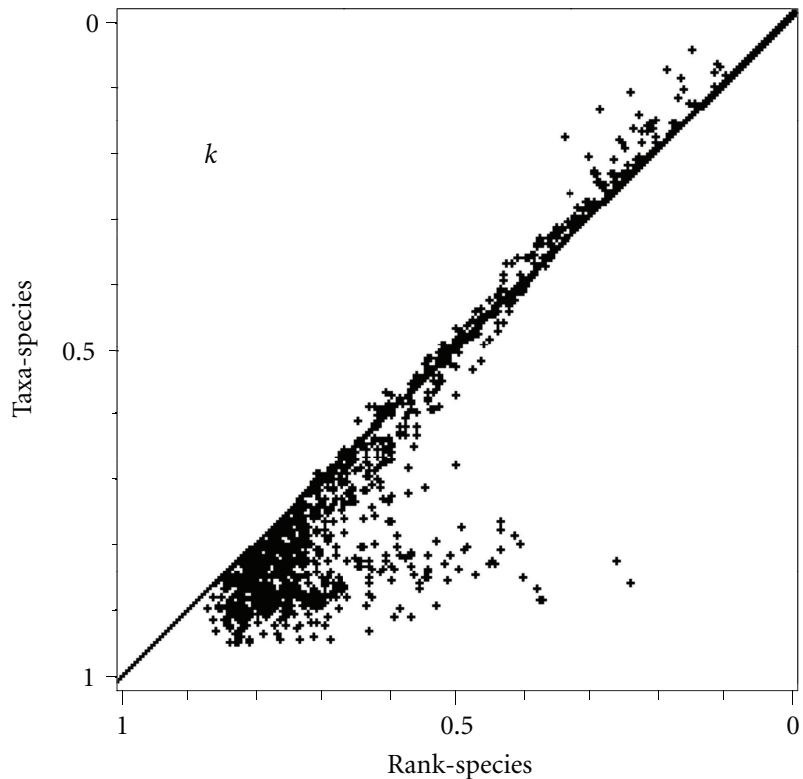

Figure 7: Comparison between the coefficient $(k)$ of geometric series based on either rank-species or taxa-species. For $8 \times 8$ matrix and presented sample points randomly reduced to $20 \%$.

The second type of models is statistical assembly models based on species arriving into an ecological domain under different scenarios of probabilities of how the species and proportions of existing species are reallocated as successive species arrive. The models include the McArthur broken stick, full random, dominance decay, new arrival, random fraction, and dominant preemptive.

I have suggested there is an equivalent third set of statistical dismantling models corresponding to a suite of species being already established but subsequently some change in conditions leading too their thinning [41]. The dismantling models considered three aspects: what were the assumed starting proportions of different species; which rank-species was selected for deletion or amalgamation to which of the other rank-species was any released domain added too. The present study could be represented as a dismantling model.

The assembly and dismantling models are statistical in that comparison with a particular set of empirical data can only be compared with the mean of outcomes of a large number of simulations of the assumed probabilistic interactions. The first three classes of models also assume the species arrive or depart in the order of their final (assembly) or initial rank order (dismantling), and thus in a sense depending on the wisdom of hindsight in determination. They also do not offer any functional ecological explanation of what the probabilities should be, for example, why the proportional reduction should be constant between successive species in the geometric series model.

There are possibly three concerns in these models as analogues of community formation. The first is that ranking is determined retrospectively from abundance so that there will always be a high negative correlation between abundance and rank. The second is that there is no definition of the species metric defining abundance and the models have been applied to everything from density of individuals, frequency, or presence in samples of various sizes, to biomass measurements. The third is that the identity of taxa-species is not retained through the different stages of simulation, that is, that their rank position and hence rank designation can change at each step. 
A fourth class of partial dynamic assembly and dismantling models retains the identity of taxa-species throughout, with various random additions or deletions made to each taxa-species total pool, with their relative rankings only determined at a final presentation stage. As a partial dynamic model the models also considered growth of existing pools as well as random additions or deletions.

Implicit in the above four classes of models is some form of difference in suitability or competitive interaction between species. In the past decade there has been investigation of a fifth class of neutral theory models which question whether those assumptions are necessary. They posit that observed patterns of relative species abundances at a local level can occur from stochastic random drift in population of species of neutral or equivalent suitability with random migration or speciation from a surrounding metapopulation and limited dispersion rates in the local level $([25,26]$ and associated papers). The focus in those approaches has been on density of species.

I have offered further three classes of particle packing assembly type models giving a possible functional explanation of the obtainable proportions [41]. These are based on representing individuals of species as circles in 2D (e.g., ground cover) or as spheres in $3 \mathrm{D}$ (e.g., volume or biomass) where competition for actual physical or conceptual space to give maximum packing determines the allowable size of species and density of individuals.

Similar consideration of what determines the frequency and maximum packing possible of different sized objects occurs in mathematics molecular chemistry, crystallography, in geology, in sediments (e.g., [71]), in engineering in aggregate packing, concrete and ceramics (e.g., [72]), in commercial packing, and in marketing resource space (e.g., [73]). The difference in the biological or ecological context is the need to also consider possible growth and mortality of individuals.

One class of those models considered highly regular geometric static packing initially defined by the maximum density of touching individuals of the largest species, which in turn defined the maximum size and density of the second species that could be fitted in the intercies, and similarly for other successive species.

Other models considered random loose packing where species differed in their potential size (= radii); their frequency of arrival; individuals retaining their initial location (e.g., as with rooted plants); inserted at their final size, or initial inserted at a small size (e.g., as seed) and allowed to grow in increments to their maximum size; displacement rules if surrounding individuals were smaller; allowable duration of individual at one size before mortality; duration of the iteration process.

The models were developed for ten species. One group of those models assumed uniform increments in potential sizes of species, while another group allowed varying sizes of different species as suggested by the geometric regular packing models. The models did not consider random tight packing where there might be reshuffling of positions to give tighter packing as might be possible analogues of animal populations.
The abundance/rank relationships from the particle packing models emphasized that the relationships were highly dependent on which metric of a species was used for abundance density, cumulative area or volume, and so forth.

Apart from the geometric regular packing model, the other particle packing models are statistical so comparison with particular empirical data can only be compared with the mean of a number of simulations as in the other model types.

A comparison with a moderately large set of empirical relative abundance data in the earlier paper had shown that the best fitting of the models had been the geometric series and log normal which had parameters fitted to each dataset [41]. However, as a general model across all datasets, most of the better fitting were from the particle packing group of models, more related to the size of individuals rather than their species designation and only occasionally from the assembly and dismantling group of models.

10.2. Analysis. The models considered and method of comparison with empirical data were the same as in the associated study [41]. There were about five hundred alternative models. The random particle packing models were based on ten species of equal decreases in potential maximum size.

The empirical data was the composite data for all samples from each of the four matrix sizes of $16 \times 16,12 \times 12,8 \times$ 8 , and $4 \times 4$. At the individual sample level the data was expressed as ranking either as rank-species or taxa-species for the ten most successful species (spA-spJ), and for the ranking of both density and biomass at each of the four harvests. The particle packing models allowed simultaneous testing of density and biomass ranking within samples as well as independently, with the biomass rankings possibly related to either the area or volume metric of the models. The fit was determined as the mean variance between the model and empirical sample rank values.

10.3. Results. The best fit was for models which had parameters directly determined from each sample. The best fitting was for the geometric series as outlined, followed by a quadratic polynomial for ten species, and much less for the log normal model. Within the geometric series group those models for an infinite number of species were slightly better than for a fixed number of species, and those based on the first five ranked species slightly better than considering all ten species. Those models were more prominent by the fourth harvest.

Initial analysis considered density and biomass measurements separately in relation to all available particle packing, assembly, dismantling and neutral models available, including the geometric series and log normal models where the parameters were fixed as the same across all samples. The variance of the fit between models and empirical data increased with decreasing matrix size and between successive harvests with, as noted earlier, the difference between the last harvest data being greater than between the earlier three harvests. The frequency of good fits with models was approximately equal expressed as rank-species or taxaspecies at the $16 \times 16$ matrix size with fits increasing in favour 
or the rank-species expression as matrix size decreased. Both effects would be expected because of the smoothing effect of a larger sample size. The variance was approximately similar for density and biomass at early harvest and large matrix size but increased more for density at smaller matrix sizes and later harvests. For the largest matrix size the variance across the twenty best-fitting models increased 1.3-2.2-fold for biomass and 2.5-6.3-fold for density.

Particle packing models featured amongst the best-fitting twenty models for both density and biomass measurements considered separately. Those will be discussed more fully shortly.

Geometric series and lognormal models with fixed parameters across all samples featured in relation to biomass measurements but not density measurements.

The assembly models also featured among the bestfitting for both density and biomass. The better were the fully random, dominant-decay, and McArthur-broken-stick models. The difference in the abundance/rank relationship between the fully random and dominant-decay model is small ([41]—Figure 9). The increasing contrast in biomass rank values in the later harvests was reflected in the $4 \times$ 4 matrix with the random-fraction and new-arrival model featuring in the third harvest and changing to the dominantpreemptive in the fourth harvest.

Dismantling models were common among the better fitting models for both density and biomass considered separately-though different models in each case. However, as indicated in the development of those models, many of them resulted in very similar abundance/rank relationships [41]. A feature of those models was the often large drop in rank value between the first and second ranked species, a similar value across middle ranks before a decrease at lesser ranks-leading to features of a log-normal relationship.

At the largest matrix size and first harvest those models had values for the fifth ranked species of c $40-60 \%$ for density and c70-85\% for biomass. At the smallest matrix size and last harvest the corresponding values were of c22-25\% for density and c $2-4 \%$ for biomass.

Neutral models only featured for density in the first harvest at the largest matrix size reflecting the similar designed densities of species at the early stage of the trial.

The more relevant comparisons were the simultaneous fit to both biomass and density rankings with the corresponding metric of 125 particle packing models with the biomass measurements related to either the area or volume metric, and density to the density metric.

The fit between empirical data and models was better for the larger matrix sizes ( $55 \%$ increase in variance between $16 \times 16$ and $4 \times 4$ matrix size) with most of the same models common to the best twenty at all sizes. Within the best twenty there was an increase in variance of $54-95 \%$ between the first and twentieth. The fit to the density component was poorer than that to the biomass component, possibly related to the initial experimental design of equal densities for all species. The fits were slightly better for sample as rank-species than as taxa-species.

The better fitting models were random packing selfthinning models-for both $3 \mathrm{D}$ and 2D. These were for ten species of uniform decreases of potential size, initially inserted in equal proportions at high densities of zero dimensions, then randomly selected for consideration of growth in unit increments if space available and depending on criteria of whether it was blocked or could displace surrounding individuals and criteria for mortality based on duration at one size ([41]_-Figure 4$)$. Within the 3D models there was a slight trend for the models with longer duration before mortality to improve across harvests. Models with the metric for area or volume had approximately similar frequency though there was also a trend for models with the metric of area to be better at the first harvest (though the swards were already fully closed) to those with the metric for volume at the last harvest. The fit for a composite 3D model is given later (Table 11).

The best of the non-self-thinning 3D random packing model with alternative arrival of a randomly selected species and a random selection of an individual for potential growth were ones with a higher frequency of arrival of potentially larger sized species and two where there was equal probability of arrival but with the species of the larger potential size allowed greater duration at one size before mortality ([41] Figure 4). The fit for the one with the higher arrival frequency of larger species is given later (Table 11).

There was one example of the geometric regular packing models defining sizes, densities, and other metrics of species in the best twenty for the last harvest but several in the next twenty. The best was for $3 \mathrm{D}$ square pacing of the largest in two embedded layers ([41]—Figure 6 for D3 sec) (Table 10-IV). This was followed closely by 3D single layer square packing, single layer square packing with only ground touching individuals (Table 11), single layer triangular packing, and infinite layer square packing ([41] Figure 6).

10.4. Discussion. Experimental design of the study should have been a good test of self-thinning particle-packing or dismantling models of random initial species proportions. The seven self-thinning particle packing models were generally among the best of the forty-five random and regular, $2 \mathrm{D}$ and $3 \mathrm{D}$ particle packing tested suggesting that further evaluation of such types of models is warranted. The interest in the particle packing models is that they offer a functional explanation of community formation with species competing for space with their proportions in both density and size determined by the maximum packing possible. No single packing model stood out as superior in the present study with both 2D and 3D models among the better fitting.

As indicated above, the dismantling models did feature among the better-fitting models when density and biomass were considered separately but not by the same models. The dismantling models assuming equal initial species proportion were slightly more common at the first harvest. But in general, for both the assembly and dismantling models, those that were random in one or more of the components tended to predominate suggesting a neutral type model.

While some of the above suite of particle packing models takes account of particular species interaction, for example, in the sense of whether the addition or removal is with the 
TABLE 10: Comparison of observed and simulated biomass and density proportions (\%) for top ten species using growth and mortality functions only.

\begin{tabular}{|c|c|c|c|c|c|c|c|c|c|c|}
\hline Species & $\mathrm{A}$ & $\mathrm{B}$ & $\mathrm{C}$ & $\mathrm{D}$ & $\mathrm{E}$ & $\mathrm{F}$ & G & $\mathrm{H}$ & $\mathrm{I}$ & $\mathrm{J}$ \\
\hline \multicolumn{11}{|c|}{ Biomass observed } \\
\hline I & 20 & 12 & 12 & 11 & 9 & 7 & 5 & 5 & 4 & 2 \\
\hline II & 27 & 18 & 11 & 11 & 8 & 7 & 5 & 5 & 3 & 1 \\
\hline III & 42 & 15 & 10 & 10 & 6 & 5 & 2 & 4 & 2 & 1 \\
\hline IV & 64 & 9 & 8 & 8 & 3 & 3 & $<1$ & 3 & 1 & $<1$ \\
\hline \multicolumn{11}{|l|}{ Fitted } \\
\hline I & 20 & 18 & 10 & 13 & 6 & 10 & 5 & 8 & 8 & 2 \\
\hline II & 29 & 18 & 8 & 13 & 5 & 8 & 2 & 7 & 7 & 2 \\
\hline III & 43 & 15 & 6 & 11 & 4 & 6 & 1 & 5 & 5 & 3 \\
\hline IV & 63 & 11 & 4 & 7 & 3 & 3 & $<1$ & 3 & 3 & 3 \\
\hline \multicolumn{11}{|c|}{ Density observed } \\
\hline I & 9 & 11 & 9 & 10 & 11 & 11 & 10 & 9 & 10 & 9 \\
\hline IV & 12 & 14 & 12 & 12 & 9 & 11 & 2 & 11 & 10 & 8 \\
\hline \multicolumn{11}{|l|}{ Fitted } \\
\hline I & 9 & 11 & 9 & 10 & 11 & 11 & 10 & 9 & 10 & 10 \\
\hline IV & 13 & 14 & 13 & 12 & 11 & 9 & 4 & 7 & 5 & 12 \\
\hline
\end{tabular}

TABLE 11: Mean percentage proportions of biomass and density of five species observed and modeled with fitted functions for growth and mortality only and for particle packing models and of allowance for conformity requirements.

\begin{tabular}{|c|c|c|c|c|c|c|c|c|c|c|c|}
\hline \multirow{2}{*}{ Species } & \multicolumn{5}{|c|}{ Biomass } & \multicolumn{5}{|c|}{ Density } & \multirow{2}{*}{ EMS } \\
\hline & A & B & $\mathrm{D}$ & G & $\mathrm{J}$ & $\mathrm{A}$ & $\mathrm{B}$ & $\mathrm{D}$ & G & $\mathrm{J}$ & \\
\hline Observed (4 harvests mean) & 40 & 14 & 11 & 5 & 1 & 10 & 12 & 11 & 8 & 9 & \\
\hline Growth and mortality only & 39 & 15 & 11 & 2 & 2 & 10 & 12 & 11 & 6 & 10 & 5.3 \\
\hline \multicolumn{12}{|l|}{ Packing models alone } \\
\hline Self-thin & 33 & 23 & 11 & 3 & $<1$ & 10 & 10 & 9 & 11 & 10 & 25.8 \\
\hline High large & 27 & 25 & 12 & 4 & $<1$ & 13 & 12 & 12 & 9 & 7 & 32.9 \\
\hline Regular & 64 & 16 & 3 & $<1$ & $<1$ & 1 & 2 & 6 & 6 & 34 & 97.5 \\
\hline \multicolumn{12}{|l|}{ Both } \\
\hline $\log / \operatorname{lin}(22 \%)$ & 39 & 15 & 8 & 3 & 3 & 13 & 13 & 7 & 5 & 12 & 9.0 \\
\hline Self-thin $(18 \%)$ & 42 & 20 & 11 & 2 & 1 & 16 & 14 & 14 & 8 & 9 & 24.4 \\
\hline High large $(8 \%)$ & 40 & 16 & 11 & 2 & 2 & 12 & 11 & 12 & 5 & 11 & 12.5 \\
\hline Regular (8\%) & 41 & 17 & 10 & 2 & 2 & 12 & 11 & 11 & 7 & 10 & 8.2 \\
\hline
\end{tabular}

highest or lowest ranked species, they did not take account of specific interactions between different specific species. But as Table 5 results show there can be interactions.

\section{Local/Regional Distribution}

11.1. Background. Another trend in community composition is the positive correlation between the regional distribution of a species and its local abundance, that is, species which have a wide range also tend to be more abundant where it occurs within that range than for a species of more limited distribution. Russell et al. [42] and Fox and Srivastava [43] have reviewed the various hypotheses for those relationships. The local frequency tends to be linearly related to regional species diversity in the absence of local species interaction, but to be curvilinear (decreasing local frequency relative to regional frequency) if regional species frequency varies with diversity. These patterns have been mostly investigated at large spatial scales as in colonization of island or variation between small sample units at regional scales. The present study investigated it at a microsward scale.

11.2. Analysis. For consideration of the distribution/abundance relationships, plants were as taxa-species within each matrix size rather than rank-species position; regional abundance as the proportion of all samples in which a species was present for a particular matrix size; local abundance as the proportion of plants of the particular taxa within the samples in which it occurred. The results were averaged across all samples for each matrix size and are presented for three matrix sizes (Figure 8).

11.3. Results. As matrix size increased there was the expected greater frequency of samples reaching their upper bound of a regional frequency of 1.0 (conspicuously evident for the $16 \times 16$ matrix size). Other matrix sizes gave a greater 


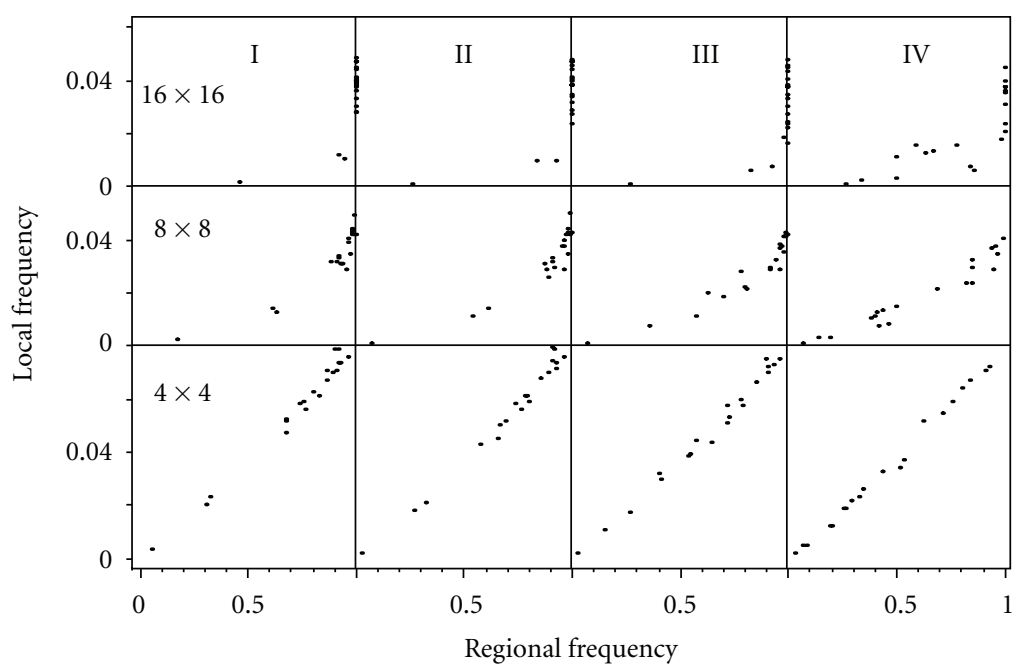

Figure 8: Mean relationship for 20 species between local frequency (proportional frequency within sample) and regional frequency (proportional occurrence across all samples) for three matrix sizes and four harvests.

dispersion of regional and local frequency values. For the nonupper band limited data the features were the strong linear relationship between local and regional frequency; the constancy of the gradient for a particular matrix size; over successive harvests, the progressive dispersion of species down that gradient.

While the reliability of mean values for each sample point is high because of the high number of subsamples involved, the variability of subsamples within a matrix size was high, for example, for the $4 \times 4$ matrix size and third harvest measurements the standard deviation between subsamples was 0.30 for regional frequency and 0.039 for local frequency. The gradient of the distribution/abundance relationship was approximately equal to the inverse of the matrix size. Using all matrix sizes from $2 \times 2$ to $12 \times 12(m=2$ to 12$)$ the best fitted relationship was

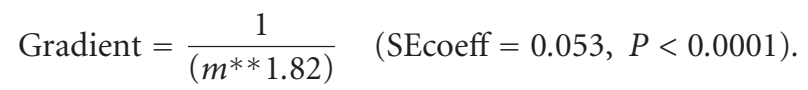

The exponential coefficient was significantly different from 2.0. The decrease in local frequency was greater than if species and plants remained randomly dispersed. A few simulation calculations suggest the greater reduction was associated with relatively uniform reduction of a species in different sample units but biased towards those resulting in new vacant samples.

11.4. Discussion. "Specie niche" and "niche width" probably contain two aspects. The first is of the combination of environmental conditions in which a species can exist and grow. The second relates to some implication about its growth potential relative to other species. In the context of the trial, all the species probably had comparable niche width in terms of adaptation to environmental factors but differed in their relative growth in the particular context.

The trial did show correlation between local and regional frequency with the strength of the relationship increasing with smaller matrix sizes as similarly reported [42]. Of the five general models reviewed, the "random placement" model was the closest analogue of the design and results of the present trial. The "sampling error" and "metapopulation dynamic" models were not appropriate. The "niche width" and "demographic" models were not appropriate in their population form but could be in their plant yield form. However, the gradient of decrease in local frequency with regional frequency as sample matrix size decreased was greater than a simple random placement model would predict.

It is suggested that the greater decrease in the gradient relates to the decreasing vigour and contribution of most species and associated greater probability of mortality resulting in vacant samples than from area and random placement alone. Thus the positive correlation between regional and local frequency was principally explainable as related to sample area as in a random placement model with possibly further decrease due to the higher probability of mortality and vacant samples for less vigorous species.

\section{Size Distribution}

12.1. Background. Loehle [39] has recently suggested that the geometric series relationship between abundance and rank is implicit from the now relatively well-established two observations of the inverse relationship between body size and density of the same species in animal and tree populations and the second observation that there is generally a very flat distribution between density and log size across individuals in a community. He demonstrated in a simulation study assuming a flat distribution between density and log size of a community with mean mass (weight) of the different species evenly spread across that scale. The same simulation suggested that the trend towards a log normal relationship was related to sampling effects in individual observations. 
12.2. Results. The aspect was investigated from the distribution of individual plant shoot weights through the four harvests. The frequency of cells within the plants present decreased through successive harvests, and the distribution of their weights became more even on a log scale (Figure 9(a)). At the left hand edge of the distribution there was a moderate to high frequency of very small plants, a decreasing frequency of slightly greater sized plants (with some classes of zero frequency), before the increasing frequency in the main distribution. These could be interpreted as seedlings still dependent on seed reserves.

Disregarding those, the kurtosis of the main region of the frequency distributions was -1.28 at the first harvest and decreased (sizes became more even) in the subsequent three harvests with successive values being $-0.52,-1.04$, and -1.35 .

The difference in the means between species became more dispersed through successive harvests (Figure 9(b)). While most taxa-species retained their relative ranking through successive harvests, some increased (species N, T, and $\mathrm{P}$ and displaced slightly upwards in figure), while other decreased (species C, F, G, K, and L). The means for the different species were not evenly spread across the relative weight range, with an increasing frequency of species means in the lower weight range.

12.3. Discussion. The present study has shown the flattening of the density/log size through successive harvests, a greater dispersion of means between species, but no indication that the species means become more evenly spread across the logweight scale.

My other study of particle packing models may be relevant [41]. Within the self-thinning group of models there was a high initial density of small propagules with subsequent growth. As iteration progressed, there was the expected decrease in density with increase in individuals mean size and variance. Where there was allowable displacement of smaller individuals by larger individuals there was a decrease in kurtosis (distribution became more even). In contrast, where there was no allowable displacement, kurtosis increased. There was no trend in kurtosis in models where there was alternate arrival and growth with density increasing.

The neutral models and the early stages of irregular sized particle packing models showed relatively flat distributions for both density and volume.

12.4. Results. The aspect was investigated from the distribution of individual plant shoot weights through the four harvests. The frequency of cells with the plants present decreased through successive harvests, and the distribution of their weights became more even on a $\log$ scale (Figure 9(a)). At the left hand edge of the distribution there was a moderate to high frequency of very small plants, a decreasing frequency of slightly greater sized plants (with some classes of zero frequency), before the increasing frequency in the main distribution. These could be interpreted as seedlings still dependent on seed reserves.

Disregarding those, the kurtosis of the main region of the frequency distributions was -1.28 at the first harvest and decreased (sizes became more even) in the subsequent three harvests with successive values being $-0.52,-1.04$, and -1.35 .

The difference in the means between species became more dispersed through successive harvests (Figure 9(b)). While most taxa-species retained their relative ranking through successive harvest, some increased (species $\mathrm{N}, \mathrm{T}$, and $\mathrm{P}$ and displaced slightly upwards in figure), while other decreased (species C, F, G, K, and L). The means for the different species were not evenly spread across the relative weight range, with an increasing frequency of species means in the lower weight range.

\section{Body Mass/Metabolic Rate}

13.1. Background. Another apparent ecological invariant across all organisms, possibly functionally responsible for the size distribution, is that for a particular temperature, metabolic rates tend to scale to the $3 / 4$ power of body mass due to the physical and geometric constraints of resource distribution networks (circulatory systems) similar within all organisms $[28,30,33]$. This was illustrated by Brown and West [32] in the common linear relationship between logarithm of body mass and logarithm of biomass productivity of mammals, birds, insects, fish, plants, and microorganisms across 18 orders of magnitude. Within that there is also a relationship related to temperature $[6,31]$. This was investigated in the present study in how the productivity in regrowth was related to previous shoot biomass.

13.2. Analysis. The analysis considered the possible applicability of the $3 / 4$ scaling relationship to the collective relative mean weight of all plants in a matrix size as a measure of body mass and the corresponding relative regrowth in a subsequent period as a measure of metabolic rate or production.

13.3. Results. For each combination of matrix size and harvest there was the expected general relationship between yield in the previous harvest and the regrowth in the subsequent period. While there was wide scatter in individual observations the trends were direct and linear-though the main interest was in the change in the gradient of that linearity for different harvest periods and matrix sizes in relation to a $3 / 4$ scaling value.

The fitted coefficient to the $\log / \log$ relationship varied linearly with both matrix size and harvest with most different from 0.75 (Figure 10). For the second and fourth harvests the smaller matrix sizes with the higher relative weights were regrowing at a proportionally faster rate than those with lower initial relative weights. As matrix size increased the trend reversed. There is no adequate explanation for the third harvest trend.

The sum of the coefficient of the abundance/rank and the reciprocal of the self-thinning relationship (Figure 9) for the third and fourth harvests of the same matrix size averaged -1.27 with a standard deviation of 0.21 . 


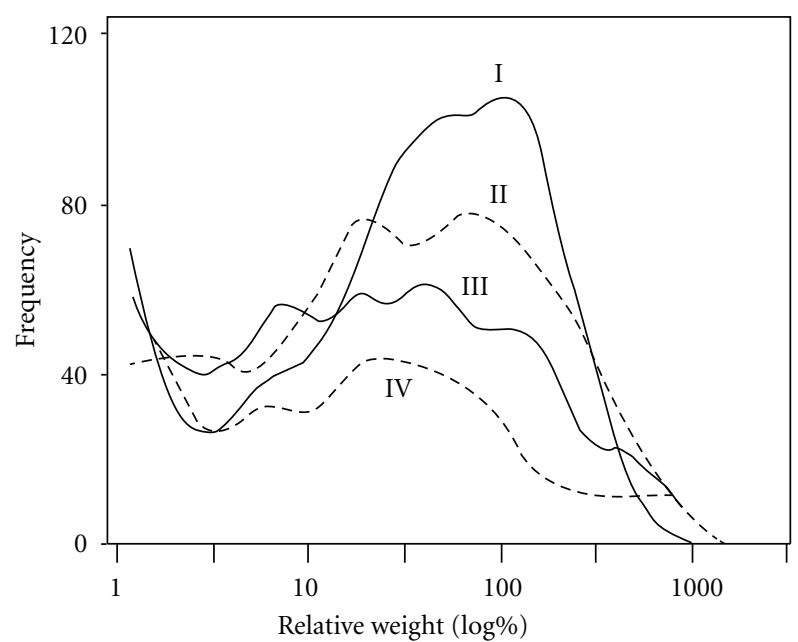

(a)

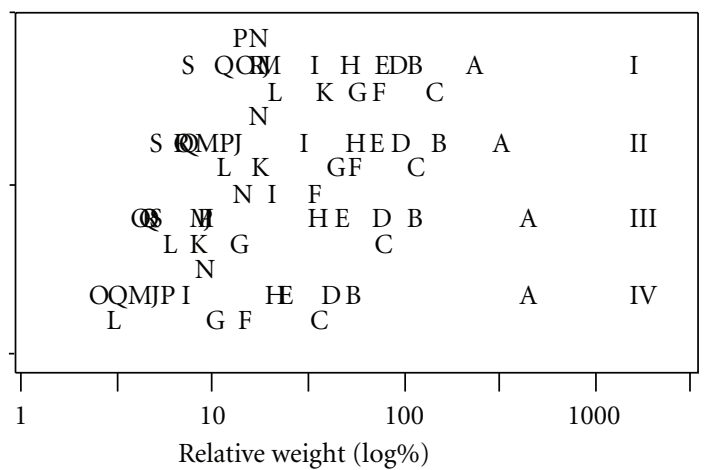

(b)

FIgURE 9: Relative frequency of different sized plants of all species at successive harvests (a) and mean for each species (b) at successive harvests for plants present.

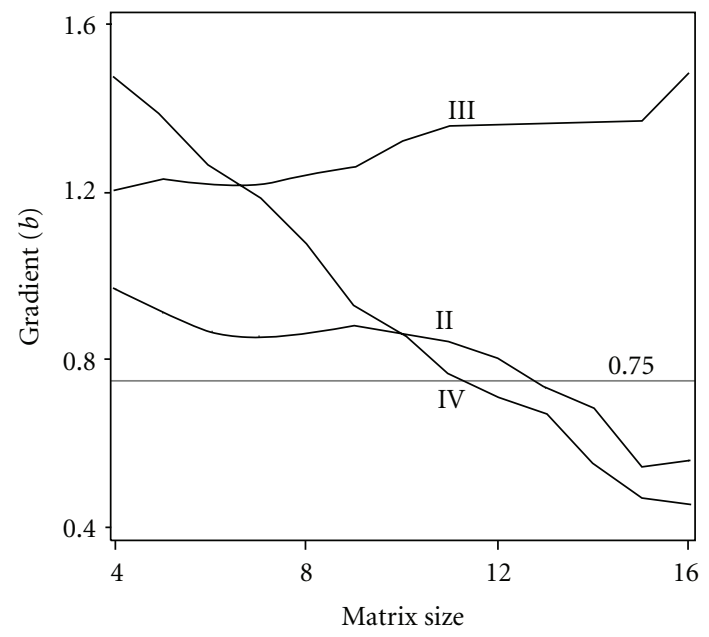

FIGURE 10: Variation in the regression coefficient between logarithm of prior weight and logarithm of regrowth rate in relation to harvest and matrix size. Standard error of estimates from c. 0.02 for group size of $4 \times 4$ to c. 0.08 for matrix size of $16 \times 16$.

13.4. Discussion. The use of previous weight and regrowth was a measure of the relationship in the present study was marginally relevant and the results inconclusive. There was a general departure from the 0.75 value. In the wider context there is probably a need to investigate whether the metabolic rates of plants vary systematically with size-as individuals or the same species, and as respiration or selfshading corrected photosynthesis. The variation between supposedly same size plants in experimental physiological or other studies is usually treated as error or random variation, but there may be an underlying trend.

\section{Size-Related Regrowth and Mortality}

14.1. Background and Analysis. The earlier results had shown that the density of species was decreasing and the interaction

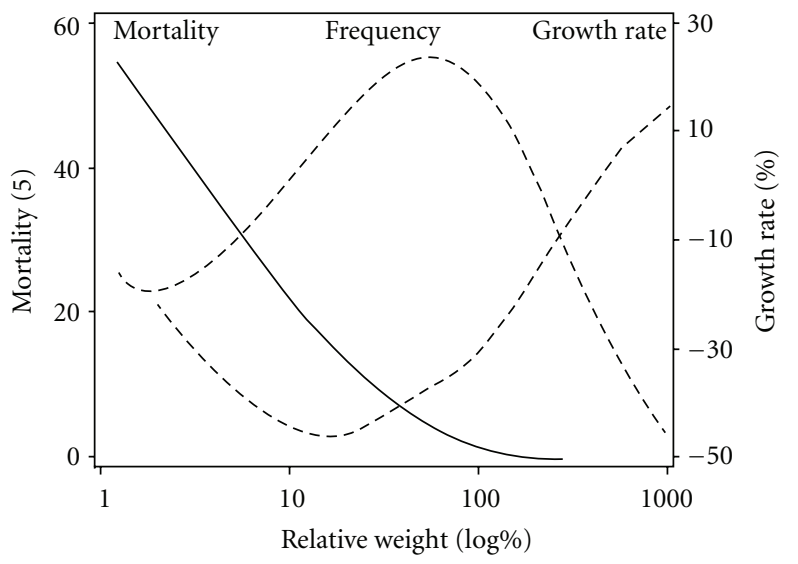

FIGURE 11: Frequency distribution across all observations of relative weights of plants (if present) at the start of a regrowth cycle $(\% \times 10)$, their mortality (\%), and regrowth rate (\%) during the subsequent cycle as related to their initial weight $(\log \%, 2=100=$ initial mean starting weight).

between plants and the self-thinning sections had indicated that it was plant size related. The general pattern for which plants died and which thrived was determined from the combined data of the frequency distribution of individual relative plant sizes at the first, second, and third harvests, and the manner in which mortality and growth rates varied in relation to that plant weight in the subsequent harvest (Figure 11). The frequencies were determined for incremental classes of relative weights on a log scale and subsequent quadratic spline line fitting.

14.2. Results. The mean distribution across all harvests in plant sizes was of a high frequency of moderate sized plants and a low frequency of some very small or large plants as had been shown for individual harvests (Figure $11 \mathrm{cf}$. Figure 9). The distribution of sizes was tending to a log normal but still somewhat right skewed. 
The mortality of plants during regrowth was greater among smaller plants and reached high levels. Within the most frequent areas of the data the decrease in mortality with plant size was near linear, but with uncertainty at either limits.

Generally for most plants their contribution was proportionally less than the total sward as shown by the mean growth rate of individual plants being generally negative. The fewer larger plants had proportionally higher growth rates. Within the bulk of the data there was an approximately positive linear relationship between the changes in growth rate with increasing plant size. The standard deviation of individual measurements around this trend was about 40 $60 \%$ for the linear section of the response. However there was a proportion of small plants whose growth rate was higher and more variable than the main trend, probably relating to the early stage when plants were of a more similar size.

For both mortality and growth rate, the trends were not limited to a few size classes and extended across three quarters of the size range. The above are generalities from the combined data. Within that there were large taxa-species differences which are enumerated as part of the subsequent simulation modelling section.

\section{Simulation Modelling}

15.1. Background. The effect of a plant size in a previous harvest on its growth or mortality in a subsequent harvest led to a consideration of whether modelling of those would be sufficient to explain changes in species proportions and mortality. There was also consideration from the particle packing models as descriptors of the abundance/rank relationship whether such overall community packing restraints might also be relevant. In an earlier simulation study I had shown that the degree to which a community may need to adhere to a $\log /$ linear relationship could explain the gradient from vegetation as a gradient in species proportions to a series of discrete communities and had introduced the concept of a "conformity index" [24]. The subsequent study of particle packing models had offered a functional reason for that conformity [41].

15.2. Analysis. The starting point of a computer simulation, like the trial, was for 20 species randomly dispersed, with each potentially present in 1200 different cells. The modelling was in three stages. The first was to simulate the results of the first harvest resulting from differences in species in germination, early establishment, and/or early mortality, mean growth rate and variability to give a range of plants of different sizes (a higher number of cells than in the empirical study were used to better simulate the later effect). The second stage was to follow the fate of each plant through a subsequent series of harvests using a fitted function for each species describing its growth or potential mortality at each harvest as related to its previous size. The third stage was to consider adjustment of initial estimates at each harvest to make them conform more closely to a packing model reflecting community interactions.
The approach was to allow for a random variation in all the parameters and to select from those the set which most closely fitted the observed sequence of species proportions and mortality. While technically it would have been possible to search among a very large set of random perturbations of parameters for the mortality and growth functions for each species, for efficiency the initial set of values was estimated from the empirical data and finer fitting determined by perturbations around those values.

For the first stage the variables for perturbation and method of simulation were as follows.

(1) Whether each of the plants of a species remained present to the first harvest from comparison of the mean probability for that species compared with a generated random uniform probability for each plant. The starting estimates for each species were values between the potential seed germination and the percentage of plants just prior to first harvest.

(2) For each of the plants that remained present, their simulated relative weight using a random normal curve estimate.

For the second stage the variables for perturbation and method of simulation were as follows.

(3) For each remaining plant of each species the probability of its death based on its relative weight in the previous period. The assumed mortality response function was a Gompertz decay curve with a lower asymptote of zero mortality at high plant weights (at least within the context of the experiment), an upper asymptote of $100 \%$ mortality at very small previous plant size, and three parameters relating to the positioning and gradient of the response function within the range of values of previous plant weight. The probability from the function was compared with a generated random uniform probability for each plant.

(4) For each of the plants remaining at each stage their relative future growth in the period was estimated from a compound unimodal Gompertz function relating their growth to previous growth. There were five parameters of the function capable of perturbation to determining upper and lower asymptotes, position, shape, and gradient of lower and upper limbs of the response function. The simulations were continued through three further harvests as corresponding with the trial. The proportion of each of the species as part of vegetation composition was determined at the end of each simulated harvest. The goodness of fit was determined as the mean variance between observed and modelled values for resulting density and biomass of species at each harvest.

(5) The process was repeated through many thousands of similar simulations with parameter values shifted to outcomes which gave successive better fits.

The third stage repeated the process with the introduction of a conformity proportion $(0-100 \%)$ relating to 
a particular packing model and constant for all species for each iteration stage. As the packing models had only been developed for ten species, these later simulations were similarly only confined to the top ten most abundant species.

(6) Density was adjusted first at each harvest estimate with a proportional increase or decrease in the number of plants of a species. These were taken as a random repeat or deletion of plants already present corresponding to the conformity proportion. The biomass weights of all plants of a species were then increased or decreased by the conformity proportion before calculation of relative species proportions.

(7) Three alternative sphere particle packing models were evaluated with species plant weights matched with sphere volumes and density with density. Of the three models used, the one with of initial high number of propagules with subsequent growth and self-thinning most closely corresponding with the trial design. The other two were for alternate random arrival and growth but with a higher frequency of arrival of potentially larger sized species; the other for regular square packing of ground-touching species. For these three the same model values were applied to all harvests.

(8) A fourth packing model only required conformity to a strict $\log /$ linear relationship but where the gradient of the relationship could vary between density and biomass measurements and at each of the harvests.

(9) The estimation of best-fitting parameters was determined by iteration as in earlier stages.

The relationship between the observed and simulated biomass and density proportions for the ten most abundant species at each of the harvests is given (Table 10). The outcomes show that a reasonable overall correspondence could be achieved between the observed and simulated values mortality and species proportion over harvests, though the fit was only modest when individual taxa-species values were examined.

That fit was obtained by using generalized mortality and growth functions with several coefficients and random search to find best-fitting values. While there was general correspondence between the observed and fitted values (Figure 12 - points and bold lines), the fit was only modest.

The variability in the observed values of mortality and regrowth rates (points) for different sized plants of each of the species was relatively large as each was based on only 120 plants, divided between mortality and regrowth measurements, and had to be further combined into size classes to get frequency estimates.

The observed mortality measurements showed the expected higher mortality of small plants and low mortality for large plants within the time-frame of the trial.

The observed mean regrowth rates differed between species, positive for $\mathrm{spA}$ (chicory), neutral for $\mathrm{spB}$ (prairie grass), and successively more negative for successively lower ranked species. Within species the regrowth rates were only weakly related to previous plant size.
The values for the parameters for the response functions for each of the species are not presented. The parameters are likely to be particular to the combination of species used in the particular conditions, so probably do not have any universality. As an example the fitted function for probability of mortality between harvests of spA (chicory) in terms of log weight (l_wt) was (in programming format) as follows:

$$
\begin{aligned}
& \text { Prob_mortality } \\
& \quad=\exp \left(-\exp \left(-6.65+\left(1 \_w t+9.18\right) * 0.46\right)\right) .
\end{aligned}
$$

And the corresponding function for its percentage regrowth was as follows:

$$
\begin{aligned}
\text { \%Regrowth }= & -20.3+144.2 \\
& *\left(\exp \left(-\exp \left(-0.46-0.173 *\left(1 \_\mathrm{wt}-18.4\right)\right)\right)\right) \\
& *\left(\exp \left(-\exp \left(-0.46+0.173 *\left(1 \_\mathrm{wt}-18.4\right)\right)\right)\right) .
\end{aligned}
$$

The construction and use of the simulation model highlighted the difference in nature of the two values estimated. The establishment and mortality estimates for each species were simulated as being independent for each species-though probably implicit that they were specific to a particular ecological context, as in this trial. By contrast the species proportions in the total vegetation are relative values where change in estimates of contribution of one species (as in its weight at one harvest) affects the relative value of all other species when they are expressed as proportions of combined total weight. The best-fitting model using only fitted growth and mortality functions achieved an error mean square for the average difference between biomass and density percentage proportions of 5.3\% (Table 11). This was considerably better than the best three particle packing models alluded to earlier. Those models were static in that the same estimates would apply to each of the four successive harvests.

The simulation also investigated whether a need for a community to conform to some community packing restraint could improve fit beyond the growth and mortality functions for individual species. The combined models were better-fitting than the particle packing models alone but were not superior to those based on growth and mortality functions alone (Table 11). The changes in the coefficients of the fitted functions were small when packing restraints were also included. The changes are given when the squarepacking ground touching regular sphere packing model was included (Figure 12—-dashed lines).

\section{General Discussion}

The results have shown that many of the features of natural ecological systems described from observations derived at a meso- or geographic scale are also present at a microscale of interaction between a group of pasture species in a small area. I did not investigate or speculate on what were the species characteristics which were the competitive basis for the difference in response between the species used in a particular 


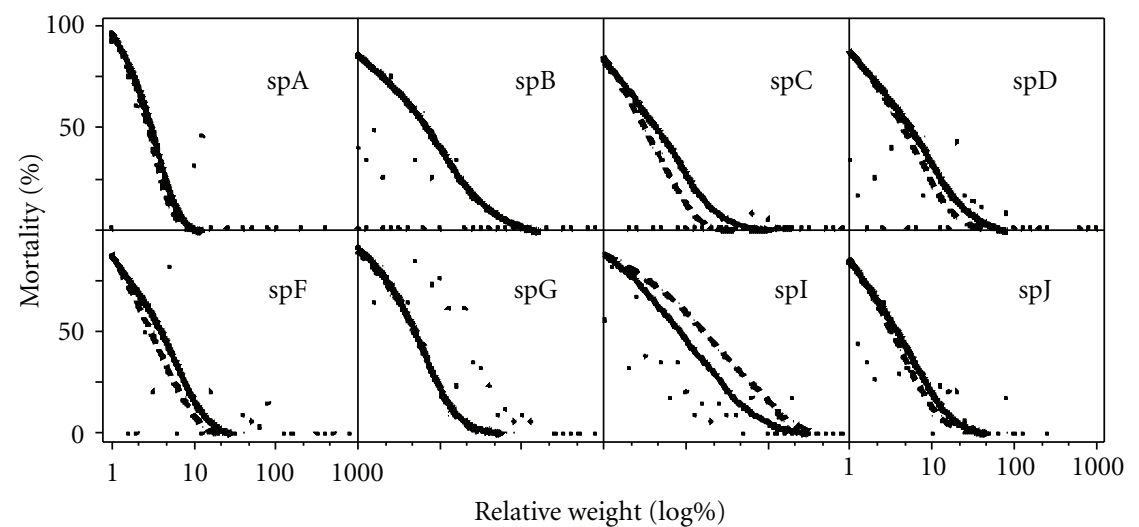

(a)

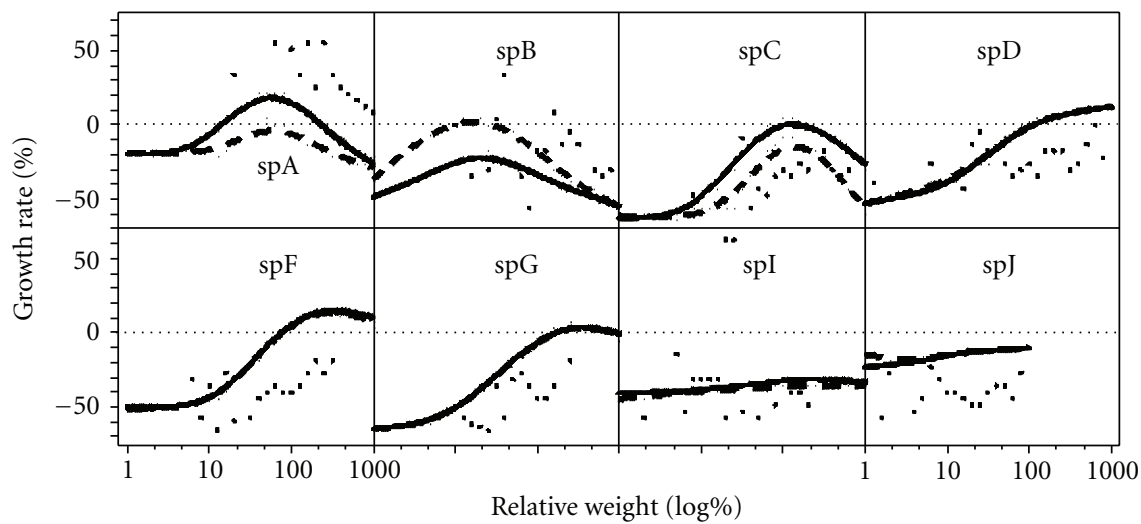

(b)

Figure 12: Comparison of observed data and simulated mortality and growth functions for eight of twenty species (1st, 2nd, 3rd, 4th, 6th, 7th, 9th, and 10th ranked taxa-species) which gave the greatest similarity with observed species mortality and vegetation composition. Points = observed, bold line, fit with only growth and mortality functions, and dashed, including conformity restraints. Coding as in Table 1 .

environmental setting and hence did not enter into the debate on physiological or morphological processes determining vegetation composition, for example, [38]. Rather it looked for features in the resulting composition patterns similar to those reported at other scales in other investigations, their possible explanations, and why they may be relatively invariant. Those patterns were present even though the trial was relatively of short term and did not include recruitment.

16.1. Productivity/Stability. Any generality between biodiversity and the productivity and/or stability of vegetation has practical importance in developing agricultural systems. The present results support the generality that productivity is more related to dominance and stability to species diversity. The sampling was from successive defoliation harvests of the same plots and thus was of severe disturbance. The generality was held under those conditions. Though initially a highly mixed sward, the trend was towards increased dominance by a few species-notably chicory (A). The second feature of the results is that while there was generality in the trends, that there was high variability of individual data around those trends.

There have been many reviews on the effects of biodiversity on ecosystem functions and these have shown that there are many different patterns and that the trends are not strong, for example, [44, 45, 56, 57, 62].

The present work showed that within the main body of the data there was little general relationship between productivity and diversity, but that the sparser surrounding data regions indicated a trend of productivity decreasing with increasing diversity (Figures 2 and 3).

Some earlier studies had emphasized the separation of productivity and stability aspects of diversity with the indication that productivity was related to dominance and stability to diversity contrary to the prevailing view $[44,45]$.

Pasture studies have indicated that biodiversity/productivity/stability relationships are present but variable. The most compelling is the 1862-1991 records from the Rothamsted Park Grass Experiment [46-48, 61]. There, diversity had a strong negative correlation with productivity (hay yield), which, if reversed, implied a strong positive relationship between dominance (fewer species) and productivity. There was also a negative correlation between mean yield and its coefficient of variation (CV), which again, if reversed, implied either a near constant STD independent of yield, and a weakly negative relationship between stability (lower CV) and diversity. The "relation was highly significant in the year (1991) for which we have the largest sample size (34 plots), 
we tentatively conclude that biomass variability may be lower in more species-rich communities, although the effect is possibly a weak one" [46]. In New Zealand, White et al. $[74,75]$ compared pasture (under sheep grazing) on four contrasting sites and reported a negative correlation between productivity (pasture growth rates and attributed to differences in soil fertility) and diversity (number of species), again implying a positive relation with dominance (fewer species). A South Island rangeland trial compared sheep grazing capacity on 61 contrasting fertiliser and grazing management treatments on an original common multispecies sward over two decades. While there was a rapid sorting out of plant species according to the experimental treatments imposed, it did not detect any general trend between pasture species diversity and secondary production or its variability [64]. That investigation also raised the issue on how such empirical results are related to the general consideration of dominance/diversity versus productivity/stability-whether the treatments are considered as perturbations of some common environment, or whether each treatment combination is seen as a different new environment with its likely changed relationships of species suitabilities.

By contrast another temperate zone study at 8 sites compared mixtures of 1 to 16 species relevant to each site of grasses, legumes, and other herbs as functional groups, established from seed and measured once or twice for 3 years. That showed productivity (hay yield) positively correlated with increasing biodiversity, with the strength of the relationships tending to increase over time ([65]-Figure 2 ) and repeated in a slightly different form in Hooper et al. ([62]-Figure 3).

A similar study with a species pool of either mixtures of 60 species or 9 dominant species and 4 functional groups showed positive correlation between productivity (hay yield) and diversity with that relationship more pronounced in experiments using only dominant species [63].

Another feature of that work was that the same results were obtained in experiments at two different scales, with the implication that large scale patterns can be investigated by small scale experiments. That the results of the present trial were reproducing many of the features observed at a large scale, also at a micro-scale, support that view.

As increasing number of studies get reported it appears that there will be no universal pattern in relation to different vegetation types or scales, with reported relationships varying from negative to positive, hump-backed, flat, or Ushaped. However, a quantification of the effects in reports to date indicates that the trend is for primary productivity to show either a hump back or increasing with species diversity $[54,56,62,66]$. The same report also indicated that temporal stability increased with diversity. Probably the next stage is to investigate in what combination of conditions similar patterns repeat.

The other feature in most of the studies, when referenced back to the variability in the source data, shows that the relationships, of whichever supposed form, are weak (e.g., Figure 3; [4]-Figure 17(a), [46]_Figure 2, [55]-Figures 1(a) and 1(b), Figure 3(a), [58, 59]-Figure 1, [62]-Figure 3,
[63] - Figure 3). This weakness or variability in the relationships is not referred to in any of the meta-analyses.

The number of species used in the present study was high by typical developed grazing pasture standards, but moderate to low for natural system investigations from which many of the generalities have developed. All the species used in the present trail would be regarded as desirable or suitable pasture components.

Some have argued that in manipulative experiments the decrease in diversity associated with the increase in productivity through the likes of fertiliser addition is related to the loss of species associated with the previous condition and the lack of species associated with the new environment. In the present trial all species were available. In a related 27-year (to date) field trial to evaluate this niche concept a mixture of 28 pasture species was drilled into a rangeland site and then $92 \mathrm{~S}$ and $\mathrm{P}$ fertiliser and grazing management treatments imposed [64]. There was rapid initial sorting out and mortality of species in the initial two years according to the treatment imposed, and slower changes over subsequent years as the legume to grass transition varied according to the fertiliser rate. However, there was no overall relationship between plant species diversity and secondary production (sheep grazing day).

Much of the previous discussion on productivity/stability has mainly looked at the effect of diversity per se. The present study has given equivalent focus on dominance and has tended to come up with different interpretations. The present results could also have been interpreted as productivity being positively related to diversity if the results had been viewed from the diversity perspective alone but, if dominance is included, show that the greater growth of a few species has more effect than diversity.

Doak et al. [51], Tilman et al. [52], and Tilmam [53] considered variability in species size or abundance as related to temporal or environmental variability. But the logic of their discussion need not rely on those effects alone, and the variation could arise from any source, such as the variation between plants of the same species, as in this study.

They also argue that the relationship between diversity and stability arises from the propagation of variances in sampling theory-what Tilman calls the "portfolio effect." Tilman illustrated this in simulation studies assuming the total biomass or productivity is equally divided among the species with their variances proportional to their contribution.

But another feature of communities is the nonoccurrence of equal contribution and the more usual log/linear relationship between abundance or productivity and rank. Insertion of such a relationship into the propagation of variances shows that the reduction in total variance is more related to dominance than diversity (Figure 13). That was based on a simulation study using different number of species and different values of the coefficient $(k)$ for a geometric series relationship between the relative abundance of different species, a square power relationship between variance and mean abundances, and a constant sum. The simulation shows that the total variance is relatively insensitive to the diversity of species after consideration of the first ranked 
species or the first few ranked species. It is only at the left margin when species are approaching equal contribution that there is marked change in total variance with diversity.

The simulation did not give any insight as to why a geometric series or other like log/linear relationship could have merit from total variance considerations or total productivity.

The simulation studies partly depend on the assumed relationship between abundance of a species and its variance, and the scaling factor between the two in the relationship var $=a^{*}$ mean** $z$. Tilman [53] argues that $z$ has values between 1 and 2 from theoretical considerations and between 1.2-1.4 (sic ? $\sqrt{2})$ in his field studies. In the present study the values were generally higher.

The possible dominance and diversity effects are not independent as they contain both a sample size effect (in small areas only a limited number of species is possible) and a biological effect (species exclusion by a dominant).

The present simulation study only considered species variance effects and not covariance species interactions. While the basis of such specific species covariance interactions, for example, legume nitrogen fixation or allelopathy, was not part of this investigation, the possibility of such specific interactions should preferably be considered. The sum of covariances was generally negative (Table 5 ), though positive for large matrix sizes. Tilman ([53]—Figure 4) also reported summed covariances variable though in three fields becoming less negative as diversity increased, as in the present study.

Besides experiments constructing vegetations of different species diversity, others have investigated the relationships by selective removal from established vegetation. For example, Smith and Knapp [60] selectively removed dominant or uncommon species from a tall grass prairie. While they found no general relationship between productivity (yield) and diversity, there was greatly reduced productivity if dominant species were removed, but unaffected by threefold reduction in minor species.

The current working hypothesis on the functional reasons for the productivity/diversity relationships while giving possible explanations of which species will dominate, some indication of what may be the rank ordering of other species, but little on what would be the relative abundance of species.

In any transfer of the diversity/productivity relationships to trophic energetics or geochemical considerations such as pasture production for grazing systems, the greater quantities are in the first few ranked species, and it is for those that the best estimates are needed.

There is an alternative view that the critical feature is as follows: what is the rank order of suitability of species within the available species pool in a particular situation? and that in some manner the subsequent quantitative features of the community are determined by that rank order alone. The apparent wide occurrence of the log/linear relationship between abundance and rank, the $-3 / 2$ thinning relationship (in this trial in a multi-species sward), the local/regional frequency relationship, and so forth would seem to support that view. In the "big picture"/ "top down" approach, the search is for processes or mechanisms which may be acting

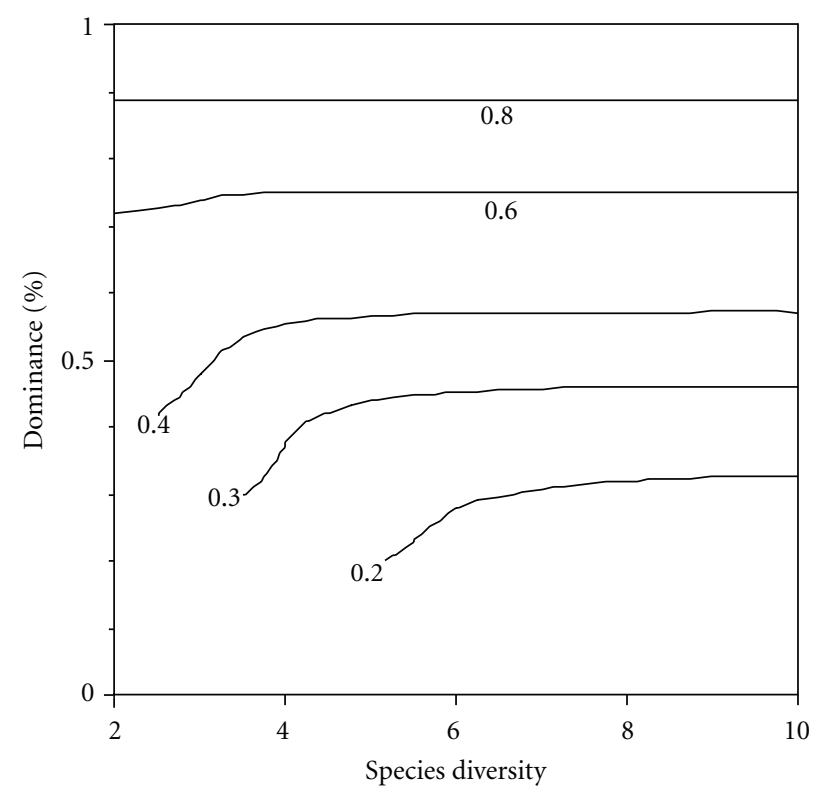

FIGURE 13: Reduction of total variance as related to both diversity and dominance.

on a group of species as a whole, on characteristics which are common to all species, but may vary in value for particular species, which would result in the common quantitative community features.

16.2. Diversity Index. The effect of dominance on total variance suggests a better definition of "diversity" (more appropriately "constancy") should relate to the distribution of abundance or mass between species rather than just the number of species per se. Hence there is some advantage in indices which combine both the dominance and species diversity aspects as in the Shannon index or the log/linear relationship. The gradient coefficient $(k)$ of the log/linear relationship depends on both the identity and rank of species, while the Shannon H' and J' do not require the later. The present work indicates that there is only marginal difference between the mean gradient coefficient based on taxa- or rank-species within sample (Figure 7). In another New Zealand pasture example from 48 sites having 3-11 species a comparison of indices relating species diversity to a soil index related to productivity the Shannon H' index gave slightly higher correlations than the number of species or Shannon J [75].

16.3. Local/Regional Frequency. "Species niche" and "niche width" probably contain two aspects. The first is of the combination of environmental conditions in which the species can exist and grow. The second relates to some implication about its growth potential relative to other contending species. In the context of the trial all the species probably had comparable niche width in terms of adaptation to environmental factors but differed in their potential relative growth in the particular context.

The present results are maybe somewhat trite in the general discussion on the relationship between local and 
regional frequency because of its microscale. The parameters were derived from the same base dataset, initial fixed densities, no recruitment, known interaction between species at a local scale (Tables 5 and 6), and the results possibly implicit in the design of the trial. But they did show a strong correlation between local and regional frequency, with the strength of the relationship increasing with smaller matrix sizes as similarly reported [42].

Of the five general models reviewed, the "random placement" model was the closest analogue to the design, duration, and results of the present trial. The "sampling error" and "meta-population dynamic" models were not appropriate. The "niche width" and "demographic" models were not appropriate in their population form but could have been in a plant yield form

However, the gradient of decrease in local frequency with regional frequency as sample size decreased was greater than a simple random model would predict. The greater decrease in the gradient related to the decreasing vigour and contribution of most species and greater probability of mortality resulting in vacant samples is suggested.

Thus the positive correlation between regional and local frequency was principally related to sample area in a random placement model, with possibly further decrease due to a higher probability of mortality and vacant samples for less vigorous species.

16.4. Interaction between Plants or Species. The implications of the observed interaction between species (Table 5) were not included in the simulation modelling (Tables 10 and 11). That would have involved both an initial random selection of species and either a random placement of plants followed by consideration of interaction with immediately surrounding plants (Table 5), or some assumption about a random allocation of the sum and distribution of covariances between species.

16.5. Self-Thinning. The empirical evidence that the $-3 / 2$ thinning rule applies over a 6 -fold difference in plant density and 9-fold differences in plant dry weight in monocultures indicates that it is an ecological invariant that needs to be explained $[2,3,5,29]$.

The present study supports the view that the selfthinning rule can also apply to mixed-species stands taken as a whole. The density and shoot weights per plant from these mixed species stands are within the same range as established for monocultures [2]. The interpretation of the present trial would be that there was little self-thinning occurring during the first two harvests but was becoming increasingly effective by the third and fourth harvests. The results for this mixed species stands should be interpreted as being consistent with the self-thinning rule rather than being a full test of the relationship.

The results were for stands of up to twenty species and plant weights considered independent of species, but using cumulative weights across successive harvest of remaining plant at a particular harvest, rather than the weights at each harvest. The applicability of the thinning rule was not investigated for individual species because of insufficient number of plants of a particular species in all but the largest matrix sizes. The trends were only becoming marked in by the third and fourth harvest with the cell size too large to demonstrate thinning in earlier harvests. The implication is that the thinning approach is relevant to the study and development of stable pasture mixtures of different species.

The empirical evidence for the self-thinning relationship between size and plant density is very compelling, with the upper thinning boundary of $-3 / 2$ seemingly explainable by either plant size/ground cover or plant allometric relationships. With the advent of considerations of the $-4 / 3$ fractal relationship relating to organisms internal circulatory systems there is probably a need for a reappraisal. It would be very difficult to differentiate between gradients of -1.5 and -1.33 in empirical data. Two of the examples used by White in 1985 in support of the then $-3 / 2$ gradient, a mixed forest and a mixed species herbaceous stand had gradients of -1.36 and -1.38 , respectively. Reanalysis of 22 animal studies indicated that $-4 / 3$ was more probable than $-3 / 2$ [7].

Westoby [2] has expressed a preference for the simpler form of the self-thinning equation as the relationship between total weight and the square root of the number of plants present $\left(\mathrm{N}^{-3 / 2}\right.$ to $\left.\mathrm{N}^{-1 / 2}\right)$. With the possibility that the relationship is a $-4 / 3$ fractal rather than $-3 / 2$ volume/area, the simpler relationship would be with the cube root of number of plants present $\left(\mathrm{N}^{-4 / 3}\right.$ to $\left.\mathrm{N}^{-1 / 3}\right)$. The present study did not detect any differences in correlation between different transformations of number of plants within each matrix size.

Reynolds and David Ford [8] suggest that the present formulation could be improved by consideration of variability in plants sizes and any point in time and of mortality and competition as related to those. That would undoubtedly be true for particular species or group of species, but for generality, the search is for features or processes common to all species even though they may need specific values for a particular group of species. The present simulation study did consider variability in plant size in relation to growth and mortality.

Westoby [2] has emphasized that plant sizes were variable in any empirical situation but that the relationships hold up using their means. The present study has also shown conformity with variability in sizes in and between species in a mixed sward. It may even suggest that it may be better to first investigate the possible spatial or environmental packing relationship of a single population of individuals of different sizes, ages, and growth rates and to only secondarily define them as different "species" according to different characteristics.

In another study I had investigated particle packing models as analogues of community formation [41]. One of those was for individuals of ten species initially inserted at high density of zero dimensions and then randomly selected for growth as spheres subject to replacement rules for displacement if growth potentially overlapped that of any other individual, with mortality if it was restrained from growing for a certain number of times. The relationships for the single species models were linear with gradients ranging from -1.71 to -1.48 and standard error of 0.02 . The relationships for the ten species models were slightly convex 
curvilinear with an average shallower gradients of -1.67 to -1.28 but -1.39 to -0.62 when large sizes predominated.

The particle packing model was in effect a competition between individuals for space with displacement rules and mortality related to duration at potential size or lack of space to grow.

Self-thinning has also been modelled as space being the resources and two coefficients representing the resource requirements of a species and the distance from which it can obtain it [4]. The study showed that it can represent most of the features of the thinning relationship but that the gradient can depart from $-3 / 2$ according to the weight given to the two coefficients.

In the present study the lack of a relationship between the gradient of the self-thinning coefficient between cumulative weight and density, and the diversity index $(k)$ of the samples from which they were obtained negates the possibility that, that is, the basis of the general similarity in the mean gradient being similar for the self-thinning and the log/linear relationship between abundance and rank.

It is noted that the self-thinning model of high initial propagules of ten species followed by growth, displacement and mortality was among the best three of the forty particle packing model evaluated.

16.6. Metabolic. The results do not support the notion of the invariance of $3 / 4$ scaling between body mass and metabolism, as it was applied in this study (Figure 10). Here it used mass and subsequent regrowth as a measure of metabolism and was applied to vegetation probably considered still in the process of developing. The results were indicating that at small matrix sizes the regrowth was larger than would be indicated by their prior mass, but that at larger matrix sizes the reverse was the case. It would seem that at small matrix sizes that already partly dominant or successful species were increasing their dominance proportionally greater, while at higher matrix sizes there was more of a balancing between species in different sectors.

There is no adequate explanation of the difference in relationship for the third harvest compared with the second and fourth harvests, unless it is part of some time trend, of some sorting-out process by the second harvest, some stability to the third harvest, and some renewal of a sortingout process as environmental condition change as indicated by the change between the irrigated and nitrogen fertiliser treatments (Table 1) though that was not being indicated by any change in relative proportion of species.

The reliability of the estimate for the coefficient of gradient of the distribution/abundance relationships being 1.82 rather than 2.0 indicates there may be something more than an inverse relationship with matrix size.

In these various studies seeking possible ecological invariants effort is generally focussed on the gradients of the linear relationships $(-3 / 2,-3 / 4,0.32$, etc. $)$. Less interest has focussed on the intercept value of those relationships and what might be the ecological significance of those values.

16.7. Simulation Model. The simulation model did achieve reasonable fit between observed and predicted proportional distributions of density and biomass between the twenty species. But that was by "trial and error" iterative fitting to each species of the coefficients of an empirical fit simple Gompertz function for mortality and a compound Gompertz function for growth. Both responses were related to plant size as expected by allometric or metabolic considerations (Figure 10). The correspondence between fit and the limited empirical data available for each species was reasonable for mortality but less than so for growth rates (Figure 11).

A simple allometric scaling (say $-1 / 4$ and $1 / 4$ ) of mortality and growth related to plant size could not be used as there was a need to have a function capable of accommodating all contingencies in actual data. There was also a need to include the more realistic option of a species having a size for optimum growth rate, not necessarily always increasing with size, and in the context of using sizes scaled to relative sizes at each harvest, that for many species it was relative negative growth.

The simulation models had not shown better fits when conformity to community packing models was added. Initially this was discouraging as it seemed to imply no community conformity requirement. However, in retrospection the effect may have been indirectly applied in estimating the mortality and growth parameters. This was indicated by the successive lowering relationship between previous growth and regrowth of the successively lower ranked species.

16.8. Experimental Procedure. If this or a similar style of experiment was to be repeated then some suggestions are made on the basis of experience with the present trial. These are advantage of initial counting out of seeds into centrifuge tubes; laying them out in the mockup of the field situation in the convenience of a laboratory prior to sowing; the delineation of plots by laying of a netting grid; a microplot size containing only one taxa-species that can be harvested in one hand-hold.

In further experiments there would be advantage in some indirect monitoring of total yield (productivity) of a treatment, by the likes of the capacitance probe or risingplate method, so that harvesting occurred at constant total yields so as to more correctly allow the amalgamation of results from different periods.

The present trial partly varied productivity independently of diversity by using the interaction of two environmental variables ( $\mathrm{N}$ fertiliser and irrigation) to get similar productivity by different methods. The concept could be extended to other environmental variables to get either similar or different productivities. An indirect method of monitoring of yield would also allow sampling of similar communities at different levels of achieved productivity or different communities needing different time periods to achieve similar yields.

The present trial did not include recruitment. That could be included by either the controlled reseeding of specific vacant cells at each harvest, or a general broadcast seeding of all species at each harvest-though that would mean checking the identity of species in all cells at each subsequent harvest. 
The present trial design could be criticized in that the samples were not independent, even though using initial random placement of individual plants, as the subsequent datasets were generated by amalgamating adjacent and overlapping groups of different sizes. This is acknowledged, and only a few statistical tests are reported. However, it is considered that the procedure did not bias the results in any systematic manner and had the advantages of creating large datasets in the search for general patterns and that any result for a particular plant, either normal or unusual, was inherent in the results from all matrix sizes. It also allowed direct investigation of the effect of sampling scale (e.g., Figure 8).

In the literature the discussion of "scale" seems to have been giving two different meanings. The first is the effect of sample or quadrat size within a particular site as it might influence allometric relationships, and the second as between sites as it might imply environmental heterogeneity.

16.9. Context. The results of the present study have been presented in the context of the vegetation component in other worldwide research for possible generalities across the functioning of different ecosystems. That had also led to investigation of packing models as a functional explanation of species proportions [41]. At the pragmatic level they were an adjunct to other studies in attempts to investigate such possible general patterns in grazing rangelands of New Zealand and elsewhere of either native grasslands or their transition towards developed pastures of generally fewer species, with hopefully the patterns having predictive or advisory value irrespective of the taxa-species involved.

The first contribution was to suggest that primary plant production should be seen as a function of the environment rather than plant species per se and related primarily to the four environmental gradients of moisture, temperature, soil fertility, and interaction with grazing animals [69]. To date the gradients have only been defined conceptually or qualitatively. The moisture gradient relates to rainfall, drainage (or irrigation), and at meso- or macroscale generally reflected in soil type. The temperature gradient reflects a metabolic feature and is derived either from direct measurements or from latitude, altitude, aspect, and slope factors usually describe as site factors. To link natural grasslands and developed pastures the soil fertility gradients considerd both natural soil fertility and applied fertiliser. The fourth gradient relates to the interaction of animals and plant growing points (grazing and treading). From cerebral overview of many reported measurements it is suggested possible to estimate potential plant production (tonne $\mathrm{DM} \mathrm{ha}^{-1} \mathrm{yr}^{-1}$ ) to $\pm 20 \%$ accuracy. The potential plant productivity is deemed to decrease logarithmical rather than linearly with reducing moisture and temperature. While the temperature scale has not yet been defined quantitatively the general relationship between temperature and metabolic rate integrated over time to give yield should provide one starting point $[6,31]$. There is as yet no suitable single parameter for the moisture gradient.

The second contribution was to use the concept of species niche or "suitability" to estimate/define where within the four environmental gradients each species would do best and how that suitability varied through the different environmental combinations. This again was/is largely a qualitative or ordinal cerebral integration based on field experience of the species, either alone or as part of possible species pools. The hope is that for a particular combination of environmental conditions a comparison of the suitability profiles of possible contending species would give their rank order of suitability. Note that for that stage it is only the rank order that is estimated. Hence the interest in the next stage is in seeing the degree to which relative contribution of species in a vegetation could be estimated from rank order alonethat is, the possible universality of the log/linear or similar packing model.

The present study only considered twenty species and would probably only be relevant to high fertility/"special purpose pasture" in New Zealand. An assessment of the suitability of a larger range of pasture species for different segments of the environmental gradients is given in [69]. The productivity/stability aspects in this study are relevant as both a generality and in the context of the change from native to developed pastures there is generally a change in the species involved and reduction in their total number. This study showed that the relationship was weak.

I speculate as a generalization that in a community of individuals (plants of different species in a vegetation) as a first approximation thatpotential primary production can be estimated from the position of a site within the gradients of environmental temperature, moisture, soil fertility and that both maximum size for the mean log-weight/density relationship, and the proportional contribution of the dominantspecies (and by implication relative proportion of other species within the available species pool) will tend to scaling values between $0.67(=2 / 3)$ and $0.75(=3 / 4)$.

The present paper has emphasised both the general trends and their variability. The variability was not considered as experimental error within a particular trial, but rather that there is real variability in those trends. The situation is probably best summarised in the verbal discussions accompanying the preparation of this paper as to its relevance to the development of pastoral systems, of whether it was about the "woods" or the "trees"-whether it was about generalities across a range of vegetations or pastures, or the search for the few more productive or desirable exceptions. The intent was to emphasize that there are probably generalities that apply to all conditions which have to be taken into consideration in the search for desirable exceptions.

\section{Acknowledgment}

The empirical field work was carried out while the author was with AgResearch, Lincoln, New Zealand, and supporting technical assistance is gratefully acknowledged.

\section{References}

[1] J. White, "Demographic factors in population of plants," in Demography and Evolution of Plant Populations, O. T. Solbrig, Ed., Blackwell, Oxford, UK, 1980. 
[2] M. Westoby, "The self-thinning rule," Advances in Ecological Research, vol. 14, pp. 167-225, 1984.

[3] J. White, "The thinning rule and its application to mixtures of plant population," in Studies on Plant Demography, J. White, Ed., pp. 291-293, Academic Press, 1985.

[4] F. R. Adler, "A model of self-thinning through local competition," Proceedings of the National Academy of Sciences of the United States of America, vol. 93, no. 18, pp. 9980-9984, 1996.

[5] H. Pretzsch, "A unified law of spatial allometry for woody and herbaceous plants," Plant Biology, vol. 4, no. 2, pp. 159-166, 2002.

[6] S. K. M. Ernest, B. J. Enquist, J. H. Brown et al., "Thermodynamic and metabolic effects on the scaling of production and population energy use," Ecology Letters, vol. 6, no. 11, pp. 990 995, 2003.

[7] C. C. Farrell-Gray and N. J. Gotelli, "Allometric exponents support A 3/4-power scaling law," Ecology, vol. 86, no. 8, pp. 2083-2087, 2005.

[8] J. H. Reynolds and E. David Ford, "Improving competition representation in theoretical models of self-thinning: a critical review," Journal of Ecology, vol. 93, no. 2, pp. 362-372, 2005.

[9] F. W. Preston, "The commonness and rarity of species," Ecology, vol. 29, pp. 254-283, 1948.

[10] Lt. Mannetje and K. P. Haydock, "The dry-weight-rank method for the botanical analysis of pastures," Journal of the British Grassland Society, vol. 18, pp. 268-275, 1963.

[11] R. H. Whittaker, "Evaluation and measurement of species diversity," Taxon, vol. 21, pp. 213-251, 1972.

[12] K. P. Haydock and N. H. Shaw, "The comparative yield method of estimating dry matter yield of pasture," Australian Journal of Experimental Agriculture and Animal Husbandry, vol. 15, pp. 663-670, 1975.

[13] J. N. G. Hargreaves and J. D. Kerr, BOTANAL a Comprehensive Sampling and Computing Procedure for Estimating Pasture Yield and Composition II. Computational Package 19, vol. 9 of Tropical Agronomy Technical Memorandum, 1978.

[14] R. M. Jones and J. N. G. Hargreaves, "Improvement to the dryweight-rank method of measuring botanical composition," Grass and Forage Science, vol. 34, pp. 181-189, 1979.

[15] R. M. May, Theoretical Ecology, Blackwell, Oxford, UK, 1981.

[16] D. L. Barnes, J. J. Odendaal, and B. H. Beukes, "Use of the dry-weight rank method of botanical analysis in the eastern Transcaal highland," Proceedings Grassland Society of South Africa, vol. 17, pp. 79-78, 1982.

[17] Y. A. M. Mansilla, M. G. Silva, F. N. Squella, C. Hullstein, and C. Wernli, "Use of the Botanal method on Medicago sativa and Dactylis glomerata pasture. III. Estimation and validation of multipliers," Avances en Produccion Animal, vol. 10, pp. 35-41, 1985.

[18] D. Scott, "The coefficients for the dry-weight-rank method of botanical analysis of pasture," Grass and Forage Science, vol. 41, pp. 319-322, 1986.

[19] D. Scott, "Constancy in pasture composition? Empirical measurements and mathematical models," New Zealand Journal of Agricultural Research, vol. 49, no. 2, pp. 183-190, 2006.

[20] H. Morlon, E. P. White, R. S. Etienne et al., "Taking species abundance distributions beyond individuals," Ecology Letters, vol. 12, no. 6, pp. 488-501, 2009.

[21] E. C. Pielou, Ecological Diversity, Wiley-Interscience, 1975.

[22] T. R. E. Southwood, Ecological Methods, Chapman \& Hall, London, UK, 2nd edition, 1978.

[23] M. Tokeshi, "Niche apportionment or random assortment: species abundance patterns revisited," Journal of Animal Ecology, vol. 59, no. 3, pp. 1129-1146, 1990.
[24] D. Scott, "Vegetation: a mosaic of discrete communities, or a continuum?" New Zealand Journal of Ecology, vol. 19, no. 1, pp. 47-52, 1995.

[25] J. Chave, "Neutral theory and community ecology," Ecology Letters, vol. 7, no. 3, pp. 241-253, 2004.

[26] M. Holyoak, M. Loreau, and D. Strong, "Neutral community ecology," Ecology, vol. 87, no. 6, pp. 1368-1369, 2006.

[27] T. M. Blackburn and K. J. Gaston, "The relationship between animal abundance and body size: a review of the mechanisms," Advances in Ecological Research, vol. 28, pp. 181-210, 1999.

[28] S. E. Cates and J. L. Gittleman, "Reading between the lines-is allometric scaling useful?” Tree, vol. 12, pp. 338-339, 1997.

[29] R. Lewin, "Ruling passions," New Scientist, pp. 34-39, 1999.

[30] B. J. Enquist and K. J. Niklas, "Invariant scaling relations across tree-dominated communities," Nature, vol. 410, no. 6829, pp. 655-660, 2001.

[31] J. F. Gillooly, J. H. Brown, G. B. West, V. M. Savage, and E. L. Charnov, "Effects of size and temperature on metabolic rate," Science, vol. 293, no. 5538, pp. 2248-2251, 2001.

[32] J. Brown and G. West, "One rate to rule them all," New Scientist, vol. 182, no. 2445, pp. 38-41, 2004.

[33] N. Marbà, C. M. Duarte, and S. Agustí, "Allometric scaling of plant life history," Proceedings of the National Academy of Sciences of the United States of America, vol. 104, no. 40, pp. 15777-15780, 2007.

[34] C. Raunkiaer, The Life Form of Plants and Statistical Plant Geography, Clarendon Press, Oxford, UK, 1934.

[35] P. Grime, J. G. Hodgson, and R. Hunt, Comparative Plant Ecology, Unwin Hymen, London, UK, 1988.

[36] J. B. Grace, "On the relationship between plant traits and competitive ability," in Perspectives on Plant Competition, J. B. Grace and D. Tilman, Eds., pp. 51-66, Academic Press, San Diego, Calif, USA, 1990.

[37] J. H. C. Cornelissen, S. Lavorel, E. Garnier et al., "A handbook of protocols for standardised and easy measurement of plant functional traits worldwide," Australian Journal of Botany, vol. 51, no. 4, pp. 335-380, 2003.

[38] J. M. Craine, "Reconciling plant strategy theories of Grime and Tilman," Journal of Ecology, vol. 93, no. 6, pp. 1041-1052, 2005.

[39] C. Loehle, "Species abundance distributions result from body size-energetics relationships," Ecology, vol. 87, no. 9, pp. 22212226, 2006.

[40] D. Ramseier and J. Weiner, "Competitive effect is a linear function of neighbour biomass in experimental populations of Kochia scoparia," Journal of Ecology, vol. 94, no. 2, pp. 305309, 2006.

[41] D. Scott, "Analogue particle and random packing models ofecological community formation," In press.

[42] L. K. Russell, S. J. Evans, L. Smith et al., "Distribution/abundance relations in a New Zealand grassland landscape," New Zealand Journal of Ecology, vol. 29, no. 1, pp. 6168, 2005.

[43] J. W. Fox and D. Srivastava, "Predicting local-regional richness relationships using island biogeography models," Oikos, vol. 113, no. 2, pp. 376-382, 2006.

[44] S. J. McNaughton, "Structure and function in California grasslands," Ecology, vol. 49, pp. 962-972, 1968.

[45] S. J. McNaughton, "Biodiversity and function of grazing ecosystems," in Biodiversity and Ecosystem Function, E.-D. Schulze and H. A. Mooney, Eds., pp. 361-383, Springer, Berlin, Germany, 1993.

[46] M. E. Dodd, J. Silvertown, K. McConway, J. Potts, and M. Crawley, "Stability in the plant communities of the Park Grass Experiment: the relationships between species richness, soil 
$\mathrm{pH}$ and biomass variability," Philosophical Transactions of the Royal Society B, vol. 346, no. 1316, pp. 185-193, 1994.

[47] J. Silvertown, M. E. Dodd, K. McConway, J. Potts, and M. Crawley, "Rainfall, biomass variation, and community composition in the park grass experiment," Ecology, vol. 75, no. 8 , pp. 2430-2437, 1994 .

[48] D. Tilman, M. E. Dodd, J. Silverton, P. R. Poulton, A. E. Johnston, and M. J. Crawley, "The Park Grass Experimentinsights from the long-term ecological study," in Long-Term Experiments in Agriculture and Ecological Sciences, pp. 287303, CAB International, 1994.

[49] UNEP, Global Biodiversity Assessment, United Nations Environment Programme, Cambridge University Press, Cambridge, UK, 1995.

[50] D. Scott, "Ecological considerations in plant invasion, with New Zealand examples," in Biological Invasion of Ecosystems by Pests and Beneficial Organisms, I. E. Yano, K. Matsuo, M. Shiyomi, and D. A. Andow, Eds., pp. 109-121, 1997.

[51] D. F. Doak, D. Bigger, E. K. Harding, M. A. Marvier, R. E. O'Malley, and D. Thomson, "The statistical inevitability of stability-diversity relationships in community ecology," American Naturalist, vol. 151, no. 3, pp. 264-276, 1998.

[52] D. Tilman, C. L. Lehman, and C. E. Bristow, "Diversitystability relationships: statistical inevitability or ecological consequence?" American Naturalist, vol. 151, no. 3, pp. 277282, 1998.

[53] D. Tilman, "The ecological consequences of changes in biodiversity: a search for general principles," Ecology, vol. 80, no. 5, pp. 1455-1474, 1999.

[54] R. B. Waide, M. R. Willig, C. F. Steiner et al., "The relationship between productivity and species richness," Annual Review of Ecology and Systematics, vol. 30, pp. 257-300, 1999.

[55] M. Loreau, S. Naeem, P. Inchausti et al., "Ecology: biodiversity and ecosystem functioning: current knowledge and future challenges," Science, vol. 294, no. 5543, pp. 804-808, 2001.

[56] G. G. Mittelbach, C. F. Steiner, S. M. Scheiner et al., "What is the observed relationship between species richness and productivity?" Ecology, vol. 82, no. 9, pp. 2381-2396, 2001.

[57] M. Rees, R. Condit, M. Crawley, S. Pacala, and D. Tilman, "Long-term studies of vegetation dynamics," Science, vol. 293, no. 5530, pp. 650-655, 2001.

[58] J. M. Chase and M. A. Leibold, "Spatial scale dictates the productivity-biodiversity relationship," Nature, vol. 416, pp. 427-430, 2002.

[59] T. Fukami and P. J. Morin, "Productivity-biodiversity relationships depend on the history of community assembly," Nature, vol. 424, no. 6947, pp. 423-426, 2003.

[60] M. D. Smith and A. K. Knapp, "Dominant species maintain ecosystem function with non-random species loss," Ecology Letters, vol. 6, no. 6, pp. 509-517, 2003.

[61] M. J. Crawley, A. E. Johnston, J. Silvertown et al., "Determinants of species richness in the park grass experiment," American Naturalist, vol. 165, no. 2, pp. 179-192, 2005.

[62] D. U. Hooper, F. S. Chapin, J. J. Ewel et al., "Effects of biodiversity on ecosystem functioning: a consensus of current knowledge," Ecological Monographs, vol. 75, no. 1, pp. 3-35, 2005.

[63] C. Roscher, V. M. Temperton, M. Scherer-Lorenzen et al., "Overyielding in experimental grassland communitiesirrespective of species pool or spatial scale," Ecology Letters, vol. 8, no. 4, pp. 419-429, 2005.

[64] D. Scott, "Sustainability of New Zealand high-country pastures under contrasting development inputs. 7. Environmental gradients, plant species selection, and diversity," New
Zealand Journal of Agricultural Research, vol. 44, no. 1, pp. 5990, 2001.

[65] E. M. Spehn, A. Hector, J. Joshi et al., "Ecosystem effects of biodiversity manipulations in european grasslands," Ecological Monographs, vol. 75, no. 1, pp. 37-63, 2005.

[66] P. Balvanera, A. B. Pfisterer, N. Buchmann et al., "Quantifying the evidence for biodiversity effects on ecosystem functioning and services," Ecology Letters, vol. 9, no. 10, pp. 1146-1156, 2006.

[67] SAS, SAS/STAT Users Guide, Version 6, SAS Institute Inc, Cary, NC, USA, 1989.

[68] SAS, SAS/GRAPH Software: Reference, Version 6, SAS Institute Inc., Cary, NC, USA, 1994.

[69] D. Scott, L. A. Maunsell, J. M. Keoghan, B. E. Allan, W. L. Lowther, and G. G. Cosssens, A Guide to Pastures and Pasture Species for the New Zealand High Country, New Zealand Grassland Association, Palmerston North, New Zealand, 1995.

[70] D. Scott, "Description of vegetation using visual ranking of species," New Zealand Journal of Ecology, vol. 12, pp. 77-88, 1989.

[71] F. J. Pettijohn, Sedimentary Rocks, Harper \& Bros, New York, NY, USA, 1975.

[72] P. Stroeven and M. Stroeven, "Assessment of packing characteristics by computer simulation," Cement and Concrete Research, vol. 29, no. 8, pp. 1201-1206, 1999.

[73] G. Péli and B. Nooteboom, "Market partitioning and the geometry of the resource space," American Journal of Sociology, vol. 104, no. 4, pp. 1132-1153, 1999.

[74] T. A. White, D. J. Barker, and K. J. Moore, "Vegetation diversity, growth, quality and decomposition in managed grasslands," Agriculture, Ecosystems and Environment, vol. 101, no. 1, pp. 73-84, 2004.

[75] T. A. White, K. J. Moore, and D. J. Barker, "The importance of local processes to landscape patterns of grassland vegetation diversity," New Zealand Journal of Agricultural Research, vol. 47, no. 2, pp. 199-207, 2004. 

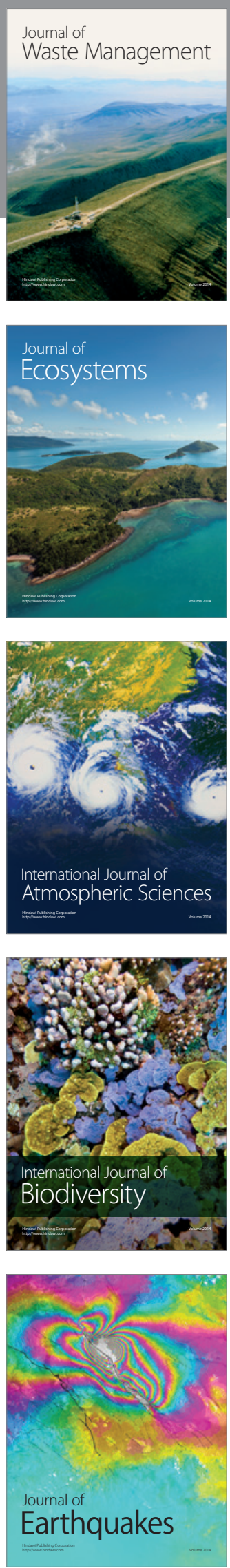
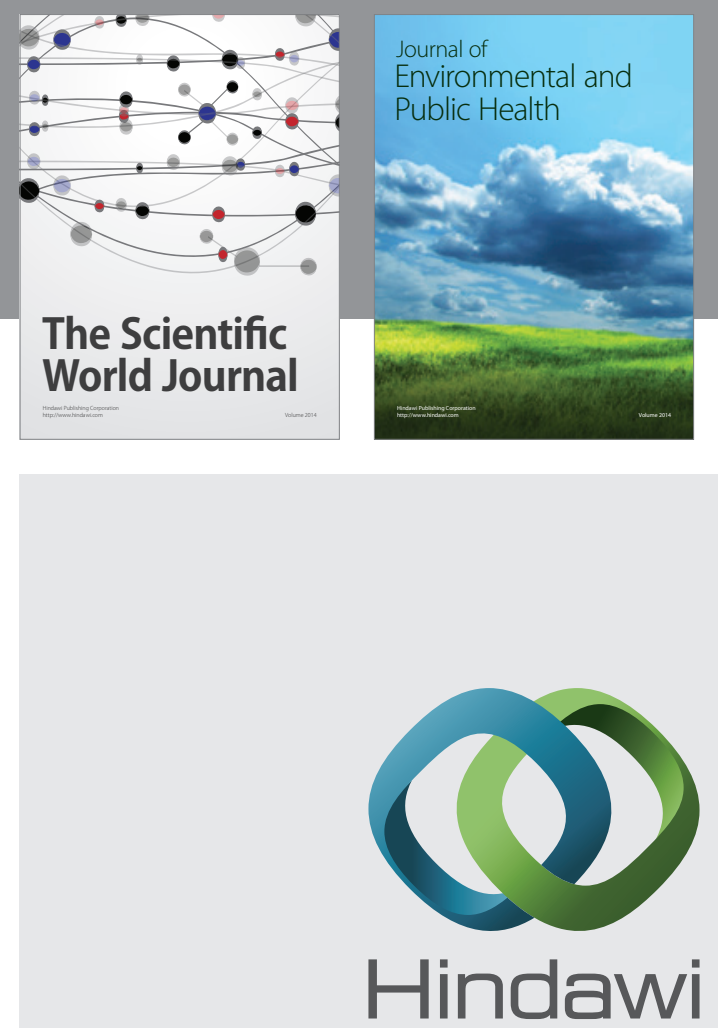

Submit your manuscripts at

http://www.hindawi.com
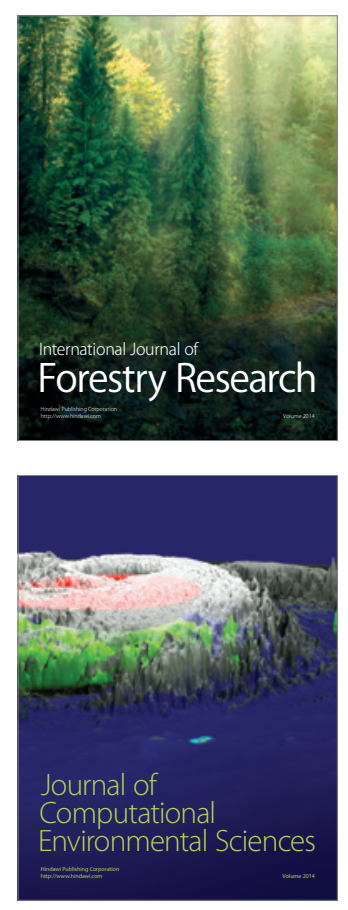
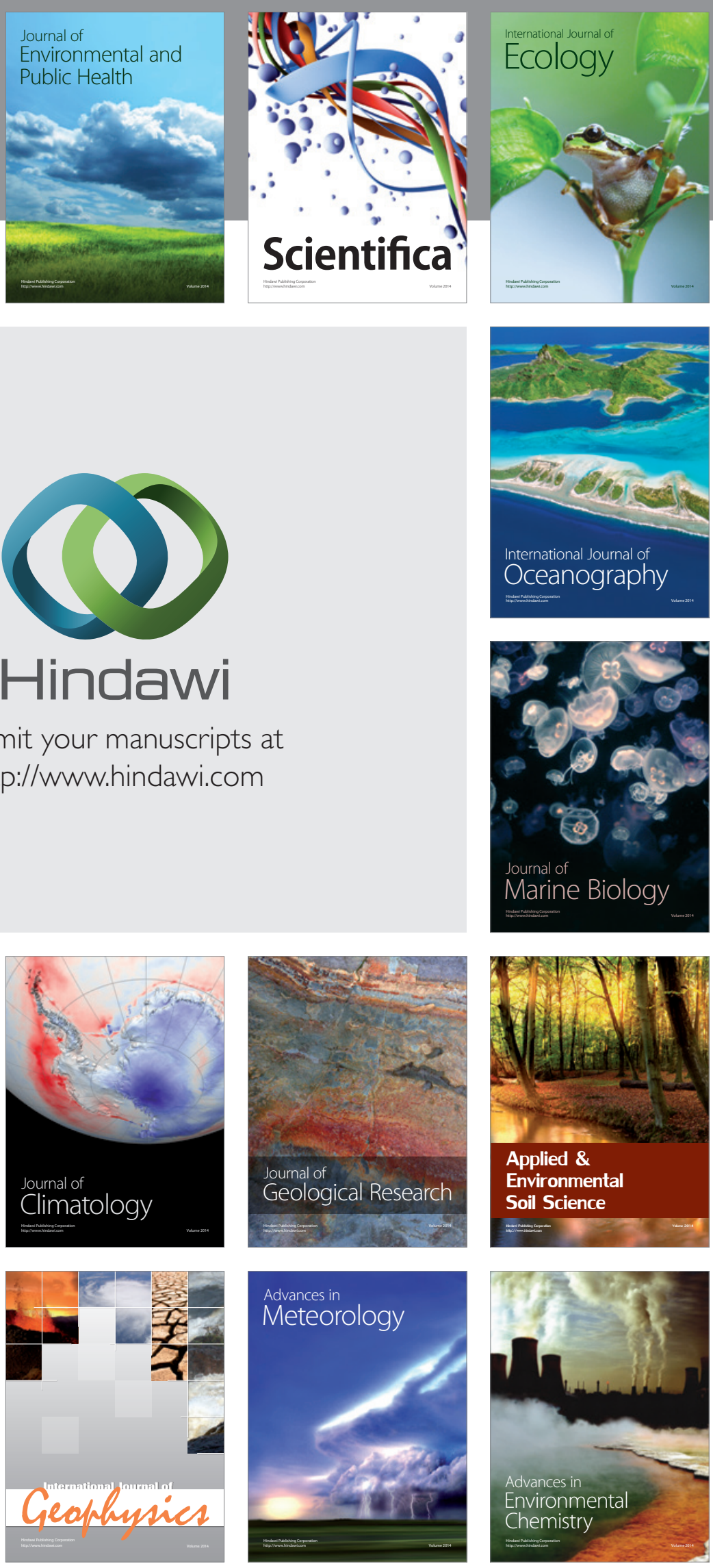